\title{
Propuesta metodológica para la determinación del mejor uso en edificios obsoletos de tejidos urbanos consolidados
}

\author{
Albert Sánchez Riera ${ }^{1}$ | Josep Roca Cladera ${ }^{2}$ \\ Recibido: 21-03-2021 | Versión final: 28-07-2021
}

Resumen

\begin{abstract}
Este artículo desarrolla una metodología en el campo de las operaciones de rehabilitación y mejora urbana en tejidos urbanos consolidados. Tiene por objeto racionalizar el proceso de adjudicación de un uso a una zona o edificio existente y pretende servir de base para edificios que actualmente han quedado en desuso u obsoletos y que, por su entidad, merecen un estudio pormenorizado del papel que podrían jugar en el contexto en que se encuentran, como generadores de nueva actividad. Así, a partir de una serie de alternativas (finitas) extraídas a partir del análisis de los equipamientos existentes en la zona de estudio, y una serie de criterios construidos mediante el análisis de indicadores (económicos, sociales y ambientales) se priorizan por secciones censales las necesidades por comparación con el resto del municipio. Para ello se propone una metodología general que combina un método de Análisis de Componentes Principales (en adelante ACP), para la extracción de indicadores que caractericen la zona, con un método multicriterio discreto conocido como Analytic Hierarchy Process (en adelante AHP), que consiga ponderar sus pesos y los de las alternativas. La metodología se ejemplifica mediante un caso de estudio de un edificio en desuso en Sabadell. A partir de la combinación de los dos métodos, y la corrección de los pesos obtenidos en función de las características de la zona, es posible establecer un mapa de necesidades para cada uso de los planteados (alternativas), y obtener, además, la mejor alternativa para la zona de estudio.
\end{abstract}

Palabras clave: Multicriterio; ACP; usos del suelo; toma de decisiones

Citación

\section{Methodological Proposal for the Establishment of the Best Use in Obsolete Buildings of Consolidated Urban Fabrics}

Abstract

\begin{abstract}
This article develops a methodology in the field of urban rehabilitation and improvement operations in consolidated urban fabrics. It aims to streamline the process of awarding an appropriate use to an existing area or building. It is intended to serve as a basis for buildings that are currently in disuse or obsolete. Due to their importance as generators of new activity, they deserve a detailed study of the role they could play in the context in which they find themselves. Thus, from a series of (finite) alternatives extracted from the analysis of the existing facilities in the study area, and a set of criteria constructed through the analysis of indicators (economic, social, and environmental), census sections needs are prioritized by comparison with the rest of the municipality. For this, a general model is proposed that combines a method of Principal Components Analysis (PCA), for the extraction of indicators that characterize the area, with a discrete multi-criteria method known as Analytic Hierarchy Process (AHP), which manages to balance their weights and the alternatives ones. The methodology is exemplified by a case study of a disused building in Sabadell. From the combination of the two methods, and the correction of the weights obtained, depending on the characteristics of the area, it is possible to establish a map of needs for each proposed use (alternatives) and to obtain the best alternative for the studied area.
\end{abstract}

Keywords: Multi-criteria; PCA; land-use; decision-making

${ }^{1}$ Dr. Arquitecto, Profesor Lector, Universitat Politècnica de Catalunya (ORCiD 0000-0003-0093-2345, Scopus Author ID: 55301158600, WoS ResearcherID: AAY-3977-2020), ${ }^{2}$ Dr. Arquitecto, Catedrático, Universitat Politècnica de Catalunya (ORCiD: 0000-0003-3970-6505, Scopus Author ID: 7101624376, WoS ResearcherID: U-2243-2019). Correo de contacto: albert.sanchez.riera@upc.edu 


\section{Introducción}

En el marco de las actuaciones locales para un desarrollo sostenible, y desde el conocimiento que todo desarrollo ha de permitir la utilización racional del territorio, en tanto que es un recurso limitado, se cree oportuno replantear nuevas estrategias de desarrollo que eviten la ocupación del suelo y la dispersión en el territorio.

El presente escrito pretende desarrollar una metodología en el campo de las operaciones de rehabilitación y mejora urbana, en tejidos urbanos consolidados, que pudiera servir de base metodológica para edificios que actualmente han quedado en desuso u obsoletos y que, por su entidad y potenciales generadores de actividad, merecen un estudio pormenorizado del papel que podrían jugar en el contexto en que se encuentran. La asignación de un uso concreto, muchas veces arbitraria y que responde a cuestiones de oportunidad política, debería ser evaluada desde variables muy diversas, ponderando su conveniencia desde un punto de vista social, pero también económico y ambiental. Pero esto no es sencillo, e implica ponderar indicadores y/o variables muy diversas y de distinta naturaleza, en muchos casos difícilmente comparables.

Se parte de la hipótesis de la no homogeneidad social, ambiental, ni económica del territorio urbano, y de que el establecimiento de un uso concreto implica en mayor o menor medida, una serie de cambios, positivos o no, en la zona donde se ubica (mejora en la calidad de vida, cohesión social, actividad económica, congestión, ruido, etc.) pero debería haber un uso que, teniendo en cuenta criterios ambientales, sociales y económicos, globalmente fuera el óptimo.

Así, a partir de una serie de alternativas (finitas) extraídas a partir del análisis de los equipamientos existentes en la zona de estudio, y una serie de criterios construidos mediante el análisis de indicadores (económicos, sociales y ambientales) este artículo intenta racionalizar el proceso de adjudicación de un uso a una zona o edificio existente, proponiendo para ello un modelo general que combina un método de análisis de componentes principales, para la extracción de indicadores de calidad, que caractericen la zona, con un método multicriterio discreto, que consiga ponderar sus pesos y los de las alternativas.

Para ello, previa la introducción y la descripción de los antecedentes y el marco teórico, se describe la metodología general empleada, ejemplificada posteriormente mediante un caso de estudio de un edificio en desuso ubicado en Sabadell. De los resultados obtenidos se concluye finalmente que, a partir de la combinación de los métodos propuestos, y la corrección de los pesos en función de las características de la zona, es posible establecer un mapa, por secciones censales, que indica la necesidad de la implantación para cada uso de los planteados (alternativas) y obtener, además, la mejor alternativa para la zona de estudio.

\section{Antecedentes y marco teórico}

Una de las formas de analizar la información proporcionada por las estadísticas y las cuentas ambientales es presentar una batería de indicadores que expresen sintéticamente una situación determinada. Los indicadores son parámetros que proporcionan información y/o tendencias sobre las condiciones y los fenómenos socioeconómicos y ambientales. Pero de manera independiente su interpretación es compleja. La importancia de disponer de indicadores de calidad o índices construidos a partir de ciertos indicadores clave (Key indicators) radica en la necesidad de proporcionar a los responsables políticos un instrumento mediante el cual se presente la información, de manera concisa y representativa, de forma que pueda ser entendida y usada fácilmente.

ACE, 16 (47) CC BY-ND 3.0 ES | UPC Barcelona, España | Propuesta metodológica para la determinación del mejor uso en edificios obsoletos de tejidos urbanos consolidados. DOI: http://dx.doi.org/10.5821/ace.16.47.10448 
Así por ejemplo al seleccionar una serie de indicadores simples como la diversidad de población, el porcentaje de delincuencia o el nivel de estudios completados, de manera individual tan solo aportan información cuantitativa, seguramente insuficiente, como herramienta para el gestor público, interesado en mejorar la calidad de vida de sus conciudadanos. Sin embargo, seguramente todos están explicando una parte de un valor que no puede medirse con unidades concretas, una variable latente que en este caso podría ser el nivel de integración social. Parece necesario pues, la construcción de estos indicadores de calidad para simplificar mediante la utilización de un modelo, los atributos y los pesos de múltiples variables e indicadores, con el objetivo de ayudar a comprender el estado de una variable determinada. No ofrecen una explicación total de la variable latente, pero bien construidos, permiten la comparación con el resto del municipio y representan una aproximación útil al estudio de ésta, ayudando a la toma de decisiones estratégicas de una manera más objetiva.

En el presente estudio, en tanto que los valores obtenidos no son índices que se puedan cuantificar, el estándar de calidad se basa en el valor de la desviación estándar de todo el conjunto de zonas evaluadas (secciones censales del municipio). Así un valor que se encuentra dentro del rango de esta desviación debe ser considerado por comparación con el resto del municipio como normal.

\subsection{Fuentes de información utilizadas}

En tanto que se pretende actuar dentro de núcleos urbanos consolidados se hace preciso un nivel de desagregación alto de los datos. El presente estudio se ha elaborado a partir de los datos socioeconómicos del censo 2001. Que, aparte de ser la mayor desagregación que permite el censo español, la utilización del censo como fuente de información socioeconómica tiene como ventaja que proporciona el mismo tipo de información para todo el territorio español, y haría posible su aplicación en otros núcleos urbanos.

Sin embargo, tiene algunas limitaciones: los datos censales pueden no caracterizar de forma completa el contexto socioeconómico porque hay un infra registro de población que corresponde principalmente a transeúntes sin residencia fija, inmigrantes extranjeros no censados y ciertos grupos marginales (Domínguez-Berjórn \& Borrell, 2005). Además, condicionado en parte por el carácter universal del censo, los datos se restringen a las principales características demográficas y a la información socioeconómica que se considera más relevante.

Aunque en este estudio se ha observado la utilidad de los datos socioeconómicos censales para la elaboración de indicadores de calidad sociales, económicos y ambientales hubiera sido deseable el acceso a indicadores más específicos y actualizados, procedentes de los datos individuales de encuestas de satisfacción como la encuesta de hogares y medio ambiente 2008, o la encuesta continua sobre presupuestos familiares. Pero los ficheros de microdatos elaborados a partir de los resultados de estas encuestas no contienen información a nivel de sección censal, tan solo municipal, siendo imposible localizar la sección donde se han encuestado a las personas.

Además, en general, se considera que los 5 años más cercanos al censo es el período más recomendable para la utilización de sus datos. Por ello, a veces se podrían plantear limitaciones para la utilización de los datos censales, ya que éstos se actualizan cada 10 años, en nuestro caso los datos utilizados están probablemente desfasados. Se ha considerado que tanto para la elaboración de los indicadores de calidad, como para la estimación de los pesos de éstos y de las alternativas, este hecho, en tanto que se trata de una aproximación metodológica, no era trascendente. Por otro lado, no se valoran intereses o factores de iniciativa privada que pudieran influir en la elección, ni otros de diferente naturaleza como podría ser la opinión pública, aunque estos podrían estar

ACE, 16 (47) CC BY-ND 3.0 ES | UPC Barcelona, España | Propuesta metodológica para la determinación del mejor uso en edificios obsoletos de tejidos urbanos consolidados. DOI: http://dx.doi.org/10.5821/ace.16.47.10448 
incorporados en otro nivel de jerarquía, como se comentará más adelante. Los resultados de esta metodología, así como la información generada pretenden servir de ayuda a la resolución del problema de decisión. No se debe extraer la conclusión de que los resultados obtenidos son una verdad absoluta por lo cual, en un informe final se debería analizar la coherencia o incoherencia de los resultados obtenidos y se deberían tener en cuenta las circunstancias y los factores que hayan influido en la metodología empleada.

\subsection{Técnicas de análisis utilizadas}

En este escrito se han combinado dos métodos de análisis de datos. El Análisis de Componentes Principales (ACP), y una técnica multicriterio conocida como AHP (Analytic Hierarchy Process), donde se ha utilizado el método Delphi con objeto de obtener una opinión fiable a partir de un conjunto de expertos (Dalkey \& Helmer, 1963).

El primero, es una técnica estadística ampliamente utilizada (Anderson, 1984; Hair et al., 1999; Johnson, 1999; Jolliffe, 1986) para la síntesis de la información, o reducción del número de variables. Es decir, ante un banco de datos con muchas variables, el objetivo será reducirlas a un menor número perdiendo la menor cantidad de información posible. Los nuevos componentes principales o factores serán una combinación lineal de las variables originales, y además serán independientes entre sí. En el caso que se presenta se utilizó esta técnica para construir los indicadores compuestos.

En relación con el análisis multicriterio, es habitual y aceptado dividir el campo de la decisión en dos grandes ramas: Discreta (DMD), que aborda problemas prácticos frecuentes, los de selección por el decisor de entre varias alternativas, pocas y lo más concretas posibles; y la rama de la Decisión multicriterio objetivo (MODM), más enfocada a problemas de diseño con alternativas no finitas que no será considerada en lo sucesivo.

Estos métodos DMD datan de los principios de los 70, y consisten básicamente, en una enumeración de alternativas y de criterios sobre los cuales se basará la decisión. En general, se deberán medir las alternativas mediante su evaluación y asignación de pesos y seleccionar aquella alternativa que mejor satisfaga las preferencias del decisor. Ahora bien, hay que tener en cuenta que, "Las alternativas deben ser completamente disjuntas y exhaustivas, es decir, no cabe concebir alternativas intermedias. Por otra parte, los criterios (o atributos, o características) sobre los cuales se basará la decisión también constituyen un conjunto discreto" (Barba-Romero, 1987). Las alternativas además habrán de ser evaluadas y normalizadas con el objeto de que puedan ser comparables con las correspondientes a otros criterios.

La literatura sobre la aplicación de métodos multicriterio en general, y para la toma de decisiones en contextos del sector público y en la planificación regional y urbana, en particular, es relativamente extensa (Davies et al., 2013; T. L. Saaty, 1990; Yager, 1988). En los últimos 20 años han proliferado numerosas aplicaciones prácticas que van desde la selección de alternativas de infraestructuras de transporte (Medina \& García, 2016) hasta la selección de proyectos estratégicos para empresas (Buchanan et al., 1999), pasando por proyectos tan diversos como la planificación de los recursos forestales (Romero, 1994) o propuestas para la clasificación de revistas (Zavadskas et al., 2014) y en definitiva múltiples proyectos en las áreas de ingeniería (Bagočius et al., 2014), la ciencia y la tecnología (Streimikiene et al., 2012; Zavadskas et al., 2015).

En el campo de la planificación territorial y urbana, sin embargo, los ejemplos son escasos a excepción quizás, de la escuela holandesa con autores como Nijkamp \& Delft, (1977) o Munda et al., (1995) que han trabajado y diversificado metodologías de análisis multicriterio especialmente en contextos de planificación regional.

ACE, 16 (47) CC BY-ND 3.0 ES | UPC Barcelona, España | Propuesta metodológica para la determinación del mejor uso en edificios obsoletos de tejidos urbanos consolidados. DOI: http://dx.doi.org/10.5821/ace.16.47.10448 
En España cabe destacar los análisis de viabilidad del transporte urbano de Zaragoza (MorenoLoscertales et al., 2019) o la aplicación de técnicas de análisis multicriterio en la evaluación de impactos ambientales en distintos campos como la conservación del suelo, la recogida selectiva, la gestión de residuos y recursos naturales, y de sistemas constructivos (García Leyton, 2004; GómezLópez et al., 2007; Lamelas García, 2014; Josa et al., 2020). Una revisión actualizada de alternativas en esta línea de evaluación de la sostenibilidad puede consultarse en (Pons-Valladares \& Nikolic, 2020).

\subsection{El problema de la estimación de los pesos}

En muchos problemas de toma de decisiones resulta necesario obtener unos pesos o indicadores de las preferencias relativas en función de unos criterios con respecto a otros. Esta estimación de preferencias relativas conlleva una fuerte carga subjetiva lo que hace necesario que para estimar dichos pesos preferenciales tengamos que interaccionar de una manera u otra con el centro decisor. La forma más sencilla de hacerlo consiste en pedir al grupo de expertos que clasifique los criterios por orden de importancia. Pero este procedimiento, aunque tiene un claro interés práctico no da información sobre la intensidad con la que el criterio i-esimo es preferido al j-ésimo. Por otra parte, ordenar simultáneamente los $n$ criterios es una tarea complicada para cualquier experto, muy especialmente cuando el número de criterios es elevado.

Existen varios procedimientos clásicos de estimación de los pesos, como el propuesto en el Método de las utilidades relativas (Fisher et al., 1959), donde se realizan estimaciones provisionales, y se van afinando para mejorar su consistencia por medio de comparaciones binarias. En la misma línea de estimación directa de los pesos cabe situar los modelos de utilidad multiatributo (UMA), exhaustivamente analizados en (Fishburn, 1990; Keeney \& Raiffa, 1993). Donde se busca expresar las preferencias del decisor sobre un conjunto de atributos o criterios en términos de la utilidad que le reporta, dentro de un contexto de la teoría de la decisión en condiciones de incertidumbre. Se trata de modelos de agregación de preferencias efectuadas respecto a criterios individuales, en los cuales se modelan las preferencias globales del decisor mediante una función de valor. Otra vía distinta es la de entropía (Zeleny, 1974), donde la idea esencial es que la importancia relativa del criterio está directamente relacionada con la información promedio de las alternativas respecto a dicho criterio y asimismo por la asignación subjetiva de la importancia que el decisor le otorgue según sus preferencias.

Un conjunto importante de métodos se basa en las Relaciones de superación o sobre calificación que utilizan el concepto de concordancia y discordancia. ( $\mathrm{S}$ b, si existen suficientes motivos a favor de a y si no se registran opiniones fuertemente contrarias a a (veto). Cuando se verifican estas dos circunstancias es posible afirmar que a supera a b. La relación de superación permite particionar y establecer un pre-orden parcial de las alternativas no dominadas del problema. Es decir, la alternativa a sobre clasifica a la alternativa $b$ (o la alternativa a es preferible a la alternativa b), cuando a es igual o superior a $b$ en una mayoría de criterios y cuando en los restantes criterios la diferencia de puntuación no es demasiado importante. El primer representante de los métodos de superación ha sido el método ELECTRE, nacido de la mano del investigador Bernard Roy (Roy, 1968) quien es considerado como un verdadero maestro de toda una generación de estudiosos de la decisión multicriterio y autoridad mundialmente reconocida en este campo. Actualmente, se conocen distintas versiones implementadas con software del método ELECTRE (I, II, III, IV, IS, TRI), que han ido enriqueciendo la metodología inicial, para permitir, de ese modo, ampliar notablemente el abanico de problemas a los que pudiera aplicarse. Amplio resumen puede consultarse en Crama \& Hansen (1983).

ACE, 16 (47) CC BY-ND 3.0 ES | UPC Barcelona, España | Propuesta metodológica para la determinación del mejor 5 uso en edificios obsoletos de tejidos urbanos consolidados. DOI: http://dx.doi.org/10.5821/ace.16.47.10448 
En general, se utiliza para reducir el tamaño del conjunto de soluciones eficientes. Funciona por bipartición, es decir, intenta dividir el conjunto eficiente en dos subconjuntos: el de las alternativas más favorables para el decisor (el núcleo) y el de las alternativas menos favorables, es decir las peores. De entre los métodos más recientes aparecidos dentro de esta categoría cabe destacar al método PROMETHEE. Su referencia pionera es Jean Pierre Brans (J.-P. Brans \& Mareschal, 2005) y en la actualidad también se dispone de diversas versiones implementadas con software del método, que han ido perfeccionando las ideas iniciales y ampliando la gama de problemas a ser tratados en la práctica. Jean Pierre Brans fue discípulo de Bernard Roy y continuador de los métodos de superación abriendo una nueva perspectiva en los mismos. A partir de estos dos grandes exponentes, los métodos ELECTRE (Figueira et al., 2005) y los métodos PROMETHEE (J. P. Brans et al., 1986), han aparecido un gran número de variantes y de métodos conexos propuestos y aplicados principalmente por estudiosos y profesionales europeos.

Otro conjunto importante y amplio de procedimientos de estimación de pesos recae en lo que podemos denominar procedimientos por aproximación. Representante significativo de este tipo de procedimientos es el conocido AHP (Analytic Hierarchy Process) (R. W. Saaty, 1987). Este método, de ayuda a la toma de decisiones, ampliamente conocido en el mundo empresarial, y que ha tenido un gran impacto a nivel teórico como práctico, permite ante un conjunto de alternativas posibles, llegar a una priorización de éstas, utilizando la comparación por pares entre elementos mediante una escala fundamental diseñada a tal efecto. Así, a partir de $n$ criterios el decisor estima una matriz $(n \times n)$, $A=[a i j]$, donde $a_{i j}$ es la medida (subjetiva) de la importancia relativa del criterio i frente al j, según una escala normalizada de 1 (igual importancia) a 9 (absolutamente más importante).

Para ello el experto ha de realizar una comparación por pares obteniendo el vector de pesos de los criterios. La comparación por pares permite al experto simplificar la ponderación a la comparación de dos casos, de manera que los juicios en principio deberían ser más fiables. Sin embargo, en la práctica el decisor cometerá ciertas inconsistencias al estimar las medidas que habrá que evaluar. La inconsistencia surge cuando algunos juicios de la matriz de comparación de pares se contradicen con otros, los miembros encuestados están poco seguros o hacen malas apreciaciones al comparar algunos de los elementos, por la falta de información, errores al tabular el dato de evaluación, etc. Desde el punto de vista del AHP, es deseable que la relación de consistencia (RC) de cualquier matriz de comparación de pares sea menor o igual a 0.10. RC = IC / IA, (T. L. Saaty, 1994) donde IC es el índice de consistencia, e IA es el Índice Aleatorio de una matriz de comparaciones pareadas, generada de forma aleatoria y que depende del número de elementos que se comparan (Frías Jiménez, 2008).

Estos dos últimos enfoques multicriterio de tipo discreto, el método ELECTRE y el método AHP, son los más utilizados en la práctica, aunque existen otros procedimientos como el lexicográfico. (Debreu, 1959; Fishburn, 1974) el de Concordancia. (Nijkamp \& Delft, 1977); comparación de alternativas, etc.

Existen en el mercado varios paquetes informáticos dedicados a la Decisión Multicriterio discreta como lo son el AIM, ELECTRE, PROMLAC, MCView, entre otros. Específicamente para el caso del AHP, se encuentran productos comerciales como: HIPRE 3+, EXPERT CHOICE Y CRITERIUM. Muchos de ellos presentan interfaces amigables para el usuario y ofrecen completos resultados y análisis de sensibilidad. Algunos de ellos permiten bajar de Internet demostraciones gratuitas.

El método del AHP, ha sido el utilizado para la estimación de los pesos en el presente escrito, su validez y utilidad parecen estar hoy fuera de toda duda.

ACE, 16 (47) CC BY-ND 3.0 ES | UPC Barcelona, España | Propuesta metodológica para la determinación del mejor uso en edificios obsoletos de tejidos urbanos consolidados. DOI: $\underline{\text { http://dx.doi.org/10.5821/ace.16.47.10448 }}$ 


\section{Metodología}

La metodología planteada parte de una serie de indicadores simples de los que se derivarán indicadores compuestos (o de calidad), y que servirán para establecer los criterios de decisión. A estos se les asignará un valor para cada zona mediante una función que compara cada sección censal con el resto de los valores del municipio. Este valor representará "la necesidad" de mejorar estos indicadores compuestos en dicha zona y se utilizarán para corregir los pesos otorgados por los expertos, obteniendo finalmente el peso de cada indicador para la zona de estudio (Figura. 1).

Figura 1 Esquema general de la metodología propuesta.

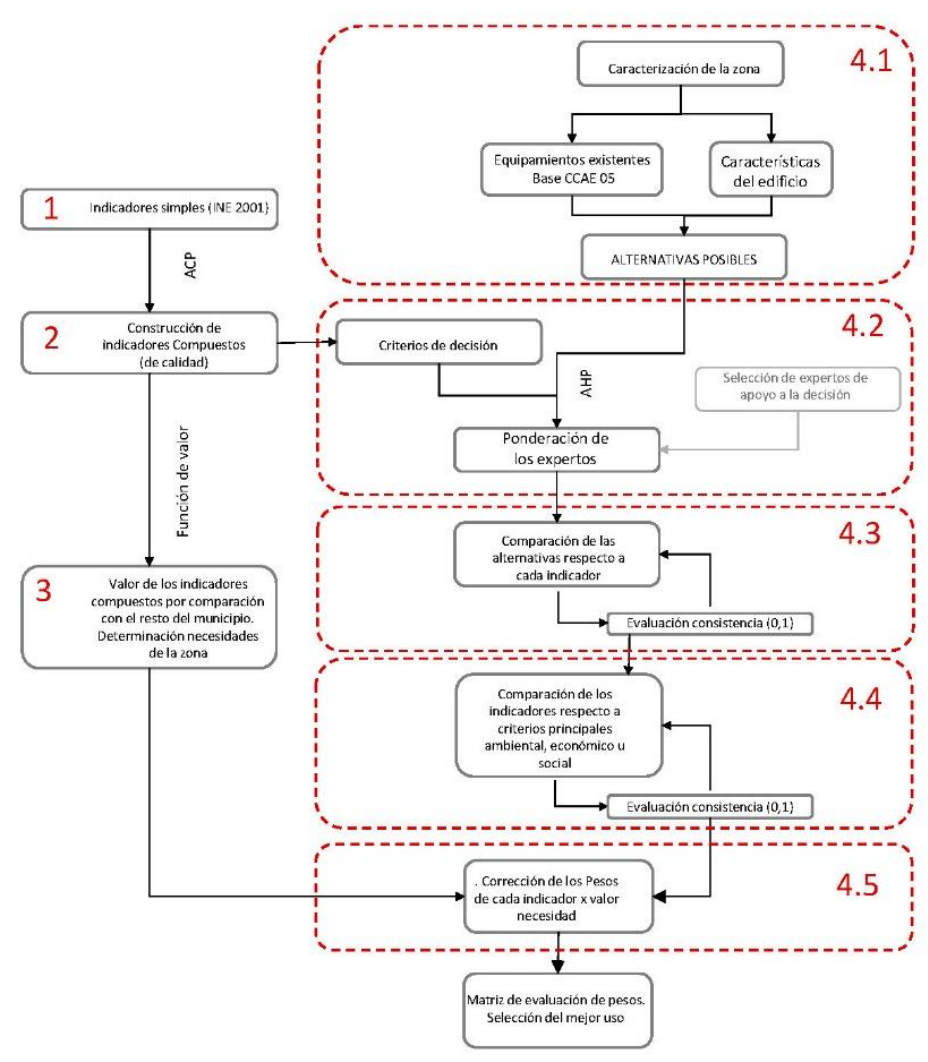

Fuente: Elaboración propia.

Se observa que, la metodología se desarrolla en cuatro grandes pasos que se desarrollan en los apartados siguientes: (1) selección de los indicadores de la zona; (2) construcción del indicador de calidad; (3) aplicación de la función de valor para determinar necesidades en la zona; (4) construcción del modelo jerárquico, que se divide a su vez, en cinco subapartados.

\subsection{Selección de los indicadores de la zona}

Dado que gran parte del interés del presente estudio es calificar y organizar jerárquicamente las secciones de acuerdo con indicadores compuestos (nivel II), para su ponderación posterior, se hace necesaria la utilización de técnicas de análisis multivariante para sintetizar un conjunto de variables relacionadas que expliquen cada una de las variables latentes. Se proponen en este caso, 6 indicadores de calidad construidos de acuerdo con los siguientes criterios: fáciles de capturar; deben 
resultar relevantes para la toma de decisiones; no deben implicar un elevado grado de dificultad en su interpretación; deben abarcar un amplio número de posibilidades.

Seis indicadores se consideraron suficientes para ejemplificar la metodología aquí planteada, sin embargo, en estudios posteriores, se hace aconsejable la construcción de un mínimo de 10 indicadores de calidad, de manera que un mayor número de variables pudieran caracterizar más detalladamente una zona. Se muestran a continuación los indicadores iniciales (Nivel I) utilizados para la construcción de los indicadores de calidad (nivel II) (Tabla 1).

Tabla 1. Composición de los indicadores construidos (Nivel II)

\begin{tabular}{|c|c|c|}
\hline & INDICADOR NIVEL । & INDICADOR DE NIVEL II \\
\hline Econ1 & Actividad de los locales (\% inactivos) & \multirow{6}{*}{ Actividad económica (iAct) } \\
\hline Econ2 & Densidad de locales (num. Locales/hogares) & \\
\hline Econ3 & Actividad de la persona ref. parados(\%parados) & \\
\hline Econ4 & Actividad pers. Ref. pensionistas (\%pensionistas) & \\
\hline Econ5 & Actividad pers. Ref. Ocupados (\%ocupados) & \\
\hline Econ6 & Tasa de población empresarial y profesional (\%) & \\
\hline Int1 & Diversidad de población (índice) & \multirow{5}{*}{ Integración Social (ilnt) } \\
\hline Int2 & Población con estudio completados (\%) & \\
\hline Int3 & Delincuencia o vandalismo en la zona (\% SI) & \\
\hline Int4 & Disponibilidad de vehículo (\%) & \\
\hline Int5 & Tipo de hogar. Unipersonal (\%) & \\
\hline iCal1 & Estructura familiar (hab/viv) & \multirow{7}{*}{ Calidad de la vivienda (iCal) } \\
\hline iCal2 & Densidad (m²/hab) & \\
\hline iCal3 & Antigüedad (índice) & \\
\hline iCal4 & Desocupación de la vivienda (\%) & \\
\hline iCal5 & Calefacción + refrigeración (\%) & \\
\hline iCal6 & Nivel de conservación (índice) & \\
\hline iCal7 & Viviendas accesibles (\%) & \\
\hline form1 & Estudios de tercer grado (\%) & \multirow{4}{*}{ Nivel de Instrucción (iForm) } \\
\hline form2 & Sin estudios persona de Ref.(\%) & \\
\hline form3 & primer grado (\%) & \\
\hline form4 & Conocimiento catalán (\% escribir) & \\
\hline Cong1 & Escasez Zonas Verdes (\% Si) & \multirow{5}{*}{ Congestión (iCong) } \\
\hline Cong2 & Contaminación o malos olores (\%si) & \\
\hline Cong3 & Ruidos exteriores (\%Si) & \\
\hline Cong4 & Densidad de hogares (personas/Ha) & \\
\hline Cong5 & Altura de la vivienda (núm. plantas s/r) & \\
\hline ePob1 & Índice de envejecimiento (\%) & \multirow{6}{*}{ Estabilidad Población (iEst) } \\
\hline ePob2 & Índice de Sobrenvejecimiento (\%) & \\
\hline ePob3 & Índice de infancia (\%) & \\
\hline ePob4 & Índice de Juventud (\%) & \\
\hline ePob5 & Índice de dependencia (0-15+>65/total) & \\
\hline ePob6 & Índice de Recambio generacional (15-19/60-64) & \\
\hline
\end{tabular}

Fuente: Elaboración propia.

ACE, 16 (47) CC BY-ND 3.0 ES | UPC Barcelona, España | Propuesta metodológica para la determinación del mejor uso en edificios obsoletos de tejidos urbanos consolidados. DOI: http://dx.doi.org/10.5821/ace.16.47.10448 
Se analizó como otro posible indicador ambiental (además del de congestión) el índice de salubridad, pero finalmente fue descartado por la poca representatividad y aleatoriedad de sus resultados (Tabla 2).

Tabla 2. Composición índice de salubridad (finalmente descartado)

\begin{tabular}{|l|l|l|}
\hline Salu1 & POCA Limpieza en las calles (\% SI/NO) & \multirow{3}{*}{ Salubridad (iSalu) } \\
\hline Salu2 & Falta de Aseo en la vivienda (\% Si/No) & \\
\hline Salu3 & Contaminación o malos olores (\% Si/No) & \\
\hline Salu4 & Evacuación aguas residuales (\% alcantarillado) & \\
\hline
\end{tabular}

Fuente: Elaboración propia.

\subsection{Construcción del indicador de calidad}

Para la construcción del indicador de calidad o nivel II, se realiza en primer lugar, un análisis de componentes principales entre los indicadores de nivel I agrupados según la Tabla 1. Una vez estimados los componentes principales y las tasas de contribución de estos, es preciso calificar cada una de las secciones de acuerdo con el índice que se está derivando. Para ello se utiliza una expresión general, que pondera las puntuaciones alcanzadas para cada componente principal, por la raíz cuadrada de su varianza (Peters \& Butler, 1970), según la siguiente ecuación para la construcción de indicadores:

$$
I_{m j}=\frac{\sum_{i=1}^{r} Z_{r j} \times \sqrt{\lambda_{r}}}{\sum_{i=1}^{r} \sqrt{\lambda_{r}}}
$$

Donde:

Imj representa el índice que se quiere obtener (iform, iCal, etc...) para cada sección censal j-esima

$Z_{\text {rj }}$ la puntuación del componente r-ésimo (factor) para la sección censal j-ésima

$\sqrt{ } \lambda$, la raíz cuadrada del autovalor para dicho componente, garantizando así que los componentes con una mayor varianza explicada tengan una mayor ponderación en la calificación del índice1.

Previo a la obtención del indicador compuesto se organizó la base de datos obtenida de acuerdo con el signo que teóricamente tenía cada componente. El índice obtenido para cada indicador de calidad ilustra la situación de cada sección en comparación con el resto de las zonas del municipio (Figura 5).

\subsection{Función de valor}

Los indicadores así construidos, presentarán valores y escalas, difícilmente comparables entre sí. Debido a ello se hace necesario unificar dichas escalas. Esta unificación se debería hacer a partir de una función que normalice las medidas de valores a través de una escala que variará entre 0 y 1.

Para la asignación del valor del indicador se consideró inicialmente utilizar una relación lineal, pero finalmente se eligió la forma de "s" ya que su incremento se detecta en los valores centrales, es decir en los valores alejados del rango de valor mínimo y máximo en donde se refleja una homogeneización.

\footnotetext{
${ }^{1}$ Azqueta Oyarzun \& Delacamara Andrés (2004) emplean esta técnica en la búsqueda de un indicador global de calidad ambiental.
}

ACE, 16 (47) CC BY-ND 3.0 ES | UPC Barcelona, España | Propuesta metodológica para la determinación del mejor uso en edificios obsoletos de tejidos urbanos consolidados. DOI: http://dx.doi.org/10.5821/ace.16.47.10448 
Una propuesta que facilita esta práctica surge en el entorno del proyecto MIVES, Proyecto de Investigación Científica y Desarrollo Tecnológico, MAT 2002 - 04310, del Ministerio de Educación y Ciencia, donde se desarrolla una metodología completa multicriterio, ampliamente utilizada en el campo de la sostenibilidad y la evaluación medio ambiental (Josa et al., 2020; Oses, 2017). De dicha metodología se desprende una función a partir de una única ecuación en la que variando ciertos parámetros ( $p$, C y K) quedan traducidos los valores para cada indicador a una única escala. La función de valor para la normalización de los indicadores se muestra en la ecuación:

$$
\begin{aligned}
& \mathrm{V}_{\text {ind }}=\mathrm{B} \cdot\left[1-\mathrm{e}^{-\mathrm{K}_{\mathrm{i}} \cdot\left(\frac{\left|\mathrm{X}-\mathrm{X}_{\min }\right|}{\mathrm{C}_{\mathrm{i}}}\right)^{\mathrm{P}_{\mathrm{i}}}}\right] \\
& \mathrm{B}=\left[1-\mathrm{e}^{-\mathrm{K}_{\mathrm{i}} \cdot\left(\frac{\left|\mathrm{X}_{\max }-\mathrm{X}_{\min }\right|}{\mathrm{C}_{\mathrm{i}}}\right)^{\mathrm{P}_{\mathrm{i}}}}\right]^{-1}
\end{aligned}
$$

Donde:

$X_{\text {min. }}$ es el origen de abscisas del indicador, frecuentemente igual a cero $X$ es la abscisa del indicador evaluado (variable para cada alternativa)

$\mathrm{P}_{\mathrm{i}}$ es un factor de forma que define si la curva es cóncava, convexa, recta o con forma de S, obteniéndose curvas cóncavas para valores de $P_{i}<1$, convexas o en forma de $S$ si $P_{i}>1$, y tendiendo a rectas para valores $\mathrm{P}_{\mathrm{i}} \mathrm{P}_{\mathrm{i}}=1$. Además, determina de forma aproximada la pendiente de la curva en el punto de inflexión de coordenadas $\left(\mathrm{C}_{\mathrm{i}}, \mathrm{K}_{\mathrm{i}}\right), \mathrm{C}_{\mathrm{i}}$ es el valor aproximado de la abscisa en el punto de inflexión, $\mathrm{K}_{\mathrm{i}}$ es el valor aproximado de la ordenada en el punto de inflexión

$\mathrm{B}$ es el factor que permite que la función se mantenga en el rango de valor de 0 a 1 . Donde $\mathrm{X}_{\max }$ es la abscisa del indicador que genera un valor igual a 1.

Figura 2. Ejemplo de la función para la asignación de valor a cada indicador

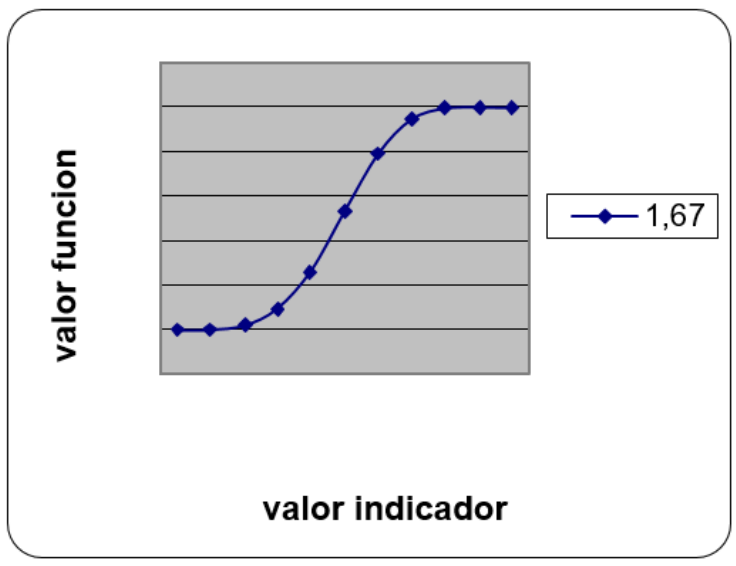

Fuente: Elaboración propia.

ACE, 16 (47) CC BY-ND 3.0 ES | UPC Barcelona, España | Propuesta metodológica para la determinación del mejor uso en edificios obsoletos de tejidos urbanos consolidados. DOI: http://dx.doi.org/10.5821/ace.16.47.10448 
Se obtendrán así valores de cada indicador de Nivel II para cada sección censal, donde los valores cercanos al máximo tendrán toda una valoración próxima a 1 y los valores cercanos al mínimo tendrán valores cercanos a 0 . De manera que se "exagera" exponencialmente una sección a partir de un determinado valor respecto a los valores medios.

Este valor representa "la necesidad" de mejora de un indicador en una zona concreta y servirá para corregir, en la zona de estudio, los pesos asignados por los expertos de manera más genérica.

\subsection{Construcción del modelo jerárquico}

Una vez obtenidos los indicadores operativos y generados los valores para cada indicador se procedió a la construcción del modelo jerárquico:

Primer paso: Identificación de alternativas (nuevos usos). Las alternativas posibles se determinaron a partir de la Clasificación Catalana de Actividades Económicas (CCAE 05). Facilitada por el área de Gestión de la información del Ayuntamiento de Sabadell. Se organizaron inicialmente por grandes grupos en base a la clasificación que realiza en INE para el colectivo de locales, en grandes áreas temáticas y son: Equipamientos de salud (ambulatorio, centro de salud, hospital...); Equipamientos educativos (colegio, facultad, guardería, escuela...); Equipamientos de bienestar social (club de ancianos, centro de servicios sociales, centro de día...); Equipamientos culturales o deportivos (teatro, cine, museo, sala de exposiciones, polideportivo...); se añade además la alternativa de zonas verdes y aparcamiento. Finalmente, la selección de alternativas que dieran respuesta a los indicadores de Nivel Il ya construidos, se realizó a partir de dos criterios de filtro: (1) De acuerdo con las características de la parcela, superficie a rehabilitar y características constructivas y tipológicas; (2) En función del número de actividades existentes en la zona, detectadas a partir de un área de influencia, en este caso 500mts, que corresponde a la zonificación de características similares obtenidas del análisis clúster inicial. Dicho análisis se realizó utilizando los indicadores compuestos de nivel II y permitió identificar zonas con características similares y poder así delimitar el ámbito de repercusión del equipamiento.

Segundo paso: Ponderación de los expertos. A partir de la selección de un grupo de expertos, a cada uno se le plantea, mediante un cuestionario, dos ejercicios: (1) Asignación del grado de importancia que otorga a cada uno de los distintos indicadores de calidad, respecto a tres criterios generales; (2) Asignación del grado de importancia que otorga a cada uso (alternativa) propuesta respecto a cada indicador de calidad. Se utilizó a tal efecto la escala del 1 al 9 (T. L. Saaty, 1994). A dos elementos de la misma importancia se les adjudica el valor 1. Cuando un elemento es extremadamente más importante que otro se le adjudica el valor 9.

El cuestionario se diseñó para ser utilizado según el método Delphi, técnica de investigación que tiene como objeto la obtención de una opinión fiable a partir de un conjunto de expertos. Es un proceso iterativo. Las preguntas se formulan de forma que se pueda realizar un tratamiento cuantitativo y estadístico de las respuestas. Así, los expertos deben ser consultados dos veces sobre la misma cuestión, de forma que puedan volver a pensar su respuesta, ayudados por la información que reciben de las opiniones (anónimas) del resto de los expertos. Todas las opiniones forman parte de la respuesta final ya que se realiza una media. (Cuestionario 1 y Cuestionario 2). Las preguntas se formulan de forma que se pueda realizar un tratamiento cuantitativo y estadístico de las respuestas (Ferri et al., 2005; Moher et al., 1999). Para la aplicación de este método se han seguido tres premisas fundamentales (Landeta, 1999): (a) Anonimato, donde la única influencia posible es la de la congruencia de los argumentos y permite que el experto puede defender sus argumentos con la tranquilidad que da saber que en caso de que sean erróneos, su equivocación no va a ser conocida por los otros expertos; (b) Iteración y realimentación controlada, ya que el cuestionario se presenta

ACE, 16 (47) CC BY-ND 3.0 ES | UPC Barcelona, España | Propuesta metodológica para la determinación del mejor uso en edificios obsoletos de tejidos urbanos consolidados. DOI: http://dx.doi.org/10.5821/ace.16.47.10448 
varias veces y como, además, se van presentando los resultados obtenidos con los cuestionarios anteriores, se consigue que los expertos vayan conociendo los distintos puntos de vista y puedan ir modificando su opinión si los argumentos presentados les parecen más apropiados que los suyos; (c) Respuesta del grupo en forma estadística, pues la información que se presenta a los expertos no es sólo el punto de vista de la mayoría, sino que se presentan todas las opiniones indicando el grado de acuerdo que se ha obtenido.

Tercer paso: Evaluación del modelo jerárquico. Se construyen las matrices de comparación de cada indicador de calidad para cada uno de los criterios seleccionados anteriormente (usos posibles). El modelo se reitera en un nivel superior construyendo las matrices de comparación de cada criterio general, en nuestro caso social, económico y ambiental, para cada uno de los indicadores de calidad anteriores. Se obtiene así el vector prioridad para cada uno de los niveles de acuerdo con el esquema siguiente (Figura 3). De manera que inicialmente se comparan las alternativas (que este caso eran los usos posibles) con cada indicador de calidad (obtenido mediante el ACP). Y en un segundo nivel jerárquico se vuelven a comparar con tres grandes criterios (ambiental, Social y económico.) a los que previamente se había ponderado su importancia.

Figura 3. Esquema jerárquico

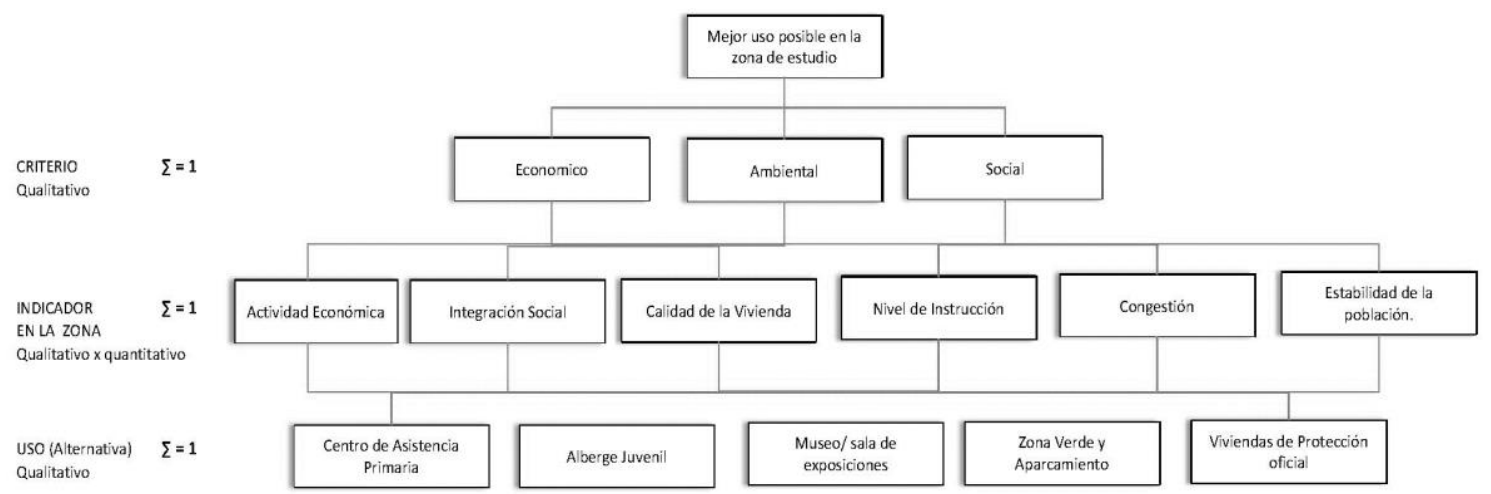

Fuente: Elaboración propia.

Esta jerarquía requiere que las evaluaciones de los expertos sean corregidas en función de las necesidades de la zona, en tanto que no es posible pretender que los expertos ponderen los pesos de los indicadores conociendo en detalle la zona de estudio. De manera que la ponderación en este nivel se hace de forma general y es adaptada posteriormente (quinto paso) por el valor de "necesidad" asignado por la función de valor. De igual forma, obtener el peso de cada elemento de manera exclusivamente cuantitativa, implicaría suponer que el peso de cada indicador es el mismo para cada criterio general. En el caso de que las respuestas de los expertos se realicen por comparaciones pareadas debe analizarse el grado de consistencia de las respuestas obtenidas y verificar que todas tengan un $\mathrm{RC}<0,1$. Tal y como se ha comentado anteriormente.

Para evitar la inconsistencia en las respuestas, la asignación de los pesos puede hacerse por valoración global, de manera que el experto debe otorgar una puntuación general a cada alternativa respecto al criterio, pero la fiabilidad de las respuestas pudiera ser menor. Para la resolución de las matrices resultantes de la comparación por pares, puede utilizarse la media geométrica por filas ${ }^{2}$ (Kaoru, 1996).

\footnotetext{
2 Entre los métodos de obtención de las prioridades locales destacan el método del auto-vector principal por la derecha (EGVM) propuesto en la versión original de AHP (Wind \& Saaty, 1980) y el método de la media geométrica
}

ACE, 16 (47) CC BY-ND 3.0 ES | UPC Barcelona, España | Propuesta metodológica para la determinación del mejor uso en edificios obsoletos de tejidos urbanos consolidados. DOI: http://dx.doi.org/10.5821/ace.16.47.10448 
Cuarto paso: Reiteración del modelo. Si bien el procedimiento puede detenerse en este punto, en tanto que se obtuvieron los valores para cada uso e indicador propuestos, el procedimiento puede aplicarse a más niveles de la estructura jerárquica; el paso siguiente en la aplicación del método AHP, consistiría en interactuar nuevamente, pero ahora en el tercer nivel jerárquico (criterios principales: social, económico y ambiental). Para ello, tendría que determinarse una relación al confrontar cada criterio principal con el objetivo final. Esta relación es la que estimará el decisor público en función de la importancia que quiere dar a cada uno de los campos o criterios principales.

Quinto paso: Corrección de los pesos. Una vez desarrollado este procedimiento se obtuvo el resumen de las ponderaciones de criterios y alternativas. Pero los pesos así obtenidos se refieren a un caso general, por el desconocimiento de los expertos sobre la zona, tal y como se ha explicado anteriormente. Para adaptar los pesos de los indicadores a la realidad social debe multiplicarse el peso del indicador por el valor otorgado por la función, para la sección censal objeto de estudio. De manera que el producto resultante represente la prioridad de cada alternativa, adaptada a la zona. (Producto de la ponderación de los expertos y el valor asignado por la función). Por ejemplo, en general la mejora de la actividad económica tiene el mayor peso desde un punto de vista de la promoción de la política económica, pero puede resultar que la zona de estudio ya revele valores altos de actividad económica con respecto al municipio, con lo cual se multiplicará por valores cercanos a 0 para corregir su peso a la baja. Es decir, a partir de una serie de indicadores simples se construyen indicadores compuestos, a los que se les asignara un valor para cada zona mediante una función que compara cada sección censal con el resto de los valores del municipio. Este valor representará la necesidad de mejorar estos indicadores compuestos en dicha zona. Y se utilizaran para corregir los pesos otorgados por los expertos a los indicadores de calidad.

Paralelamente, a partir de las características tipológicas del edificio y los equipamientos existentes en la zona, se determinan las alternativas (usos posibles) a evaluar. Que se comparan en un primer nivel respecto a cada indicador y del mismo modo en un segundo nivel, se comparan los indicadores compuestos respecto a los criterios principales: ambiental, económico y social. Esta importancia (peso) del indicador se corrige con los valores (necesidades) de la zona de estudio obteniendo finalmente el peso de cada indicador para la zona de estudio.

Finalmente deben elaborarse las matrices de evaluación obteniendo la importancia (pesos) de cada alternativa posible para la zona de estudio. El siguiente esquema presenta, en detalle, el proceso seguido en la construcción del modelo jerárquico del caso de estudio (Figura 4).

\section{Caso de estudio}

El edificio objeto de estudio se sitúa en Sabadell, Distrito 1, Sector Sant Oleguer, en el barrio de Sol i Padris. Construido en el año 1990, consta de una superficie de parcela de $1.738 \mathrm{~m}^{2}$ donde se han edificado $7.090 \mathrm{~m}^{2}$ en sótano, planta baja y 4 plantas piso. De ellos, $5.353 \mathrm{~m}^{2}$ son sobre rasante. Los usos permitidos según planeamiento son: Dominantes: plurifamiliar y residencial; Compatibles: Comercio/Oficinas y servicios, Hotelero/Restauración, Industria artesanal/Industria urbana, Almacén/Taller de reparación de vehículos/Aparcamiento, Educativo/Sanitario-asistencial, Deportivo Cultural/Asociativo/Religioso/Servicios urbanos.

por filas (RGMM), cuya utilización se ha incrementado significativamente en los últimos tiempos (Crawford \& Williams, 1985)(Aguarón \& Moreno-Jiménez, 2003).

ACE, 16 (47) CC BY-ND 3.0 ES | UPC Barcelona, España | Propuesta metodológica para la determinación del mejor uso en edificios obsoletos de tejidos urbanos consolidados. DOI: http://dx.doi.org/10.5821/ace.16.47.10448 
Figura 4. Esquema detallado del procedimiento seguido en la construcción del modelo jerárquico correspondiente al caso de estudio

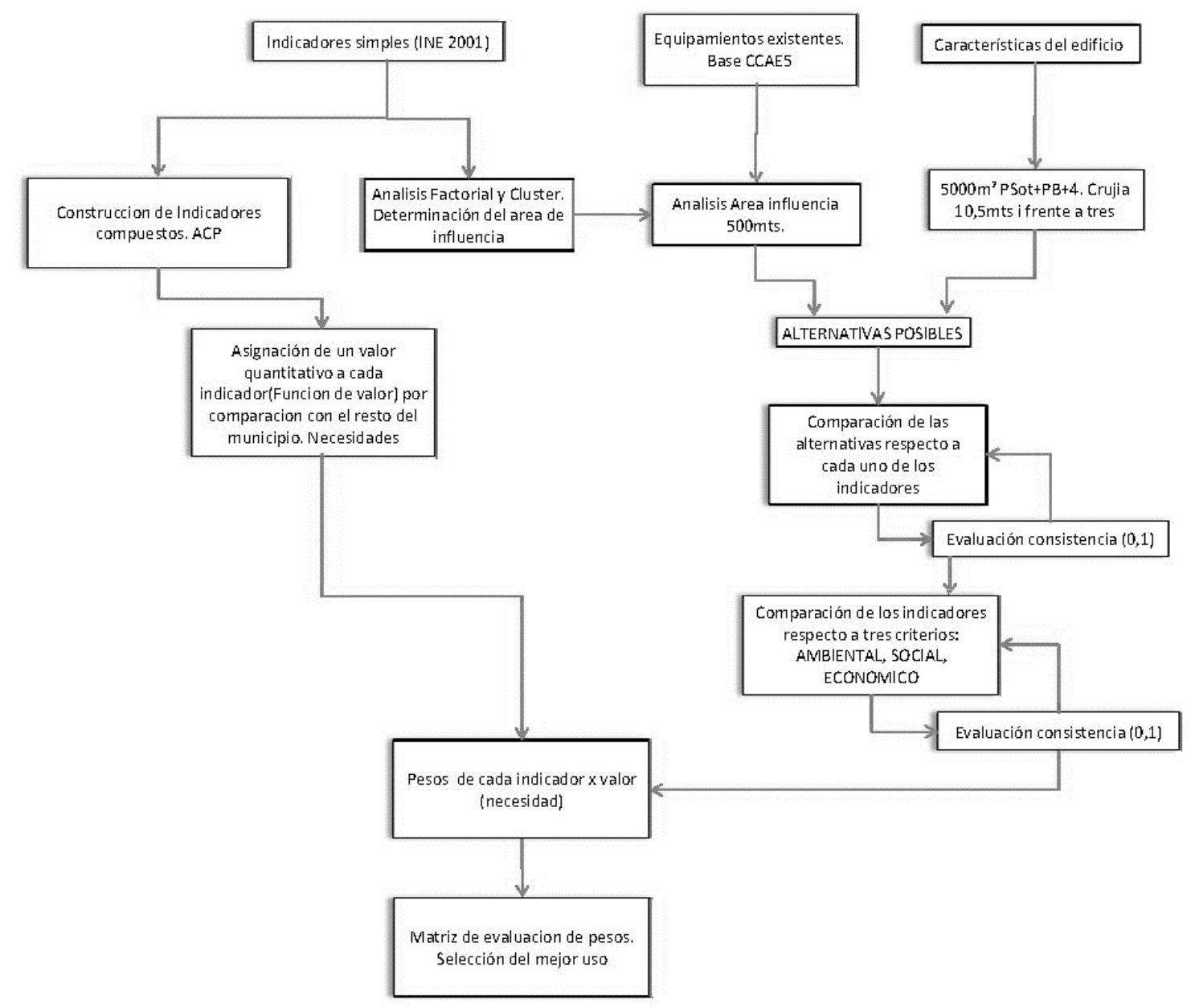

Fuente: Elaboración propia.

De acuerdo con los criterios explicados anteriormente se definieron 6 indicadores de calidad que pudieran ayudar a caracterizar la zona de estudio. (Actividad económica, Integración social, Calidad de la vivienda, Nivel de instrucción, Congestión, Estabilidad de la Población). La construcción de estos indicadores de nivel II se realizó a partir de los componentes principales extraídos del grupo de indicadores simples originales y de acuerdo con la formula anteriormente explicada (Ecuación 1).

Para la obtención de dicho indicador único se organizó la base de datos con el signo que teóricamente tenía cada componente. Con el objeto de ilustrar los resultados obtenidos, se presenta a continuación la mapificación por secciones censales de los seis indicadores compuestos (nivel II) extraídos a partir de los indicadores simples (Figura 5). Las zonas anaranjadas representan valores que están dentro de la media ( \pm desv. St.) de los valores de todo el municipio, valores en rojo representan zonas con déficits del indicador respecto al resto, es decir zonas con mayor necesidad de mejorar el valor, y en color verde se representan zonas por encima de la media (poca necesidad de mejora). 


\section{ACE Architecture, City and Environment}

Figura 5. Indicadores compuestos por sección censal
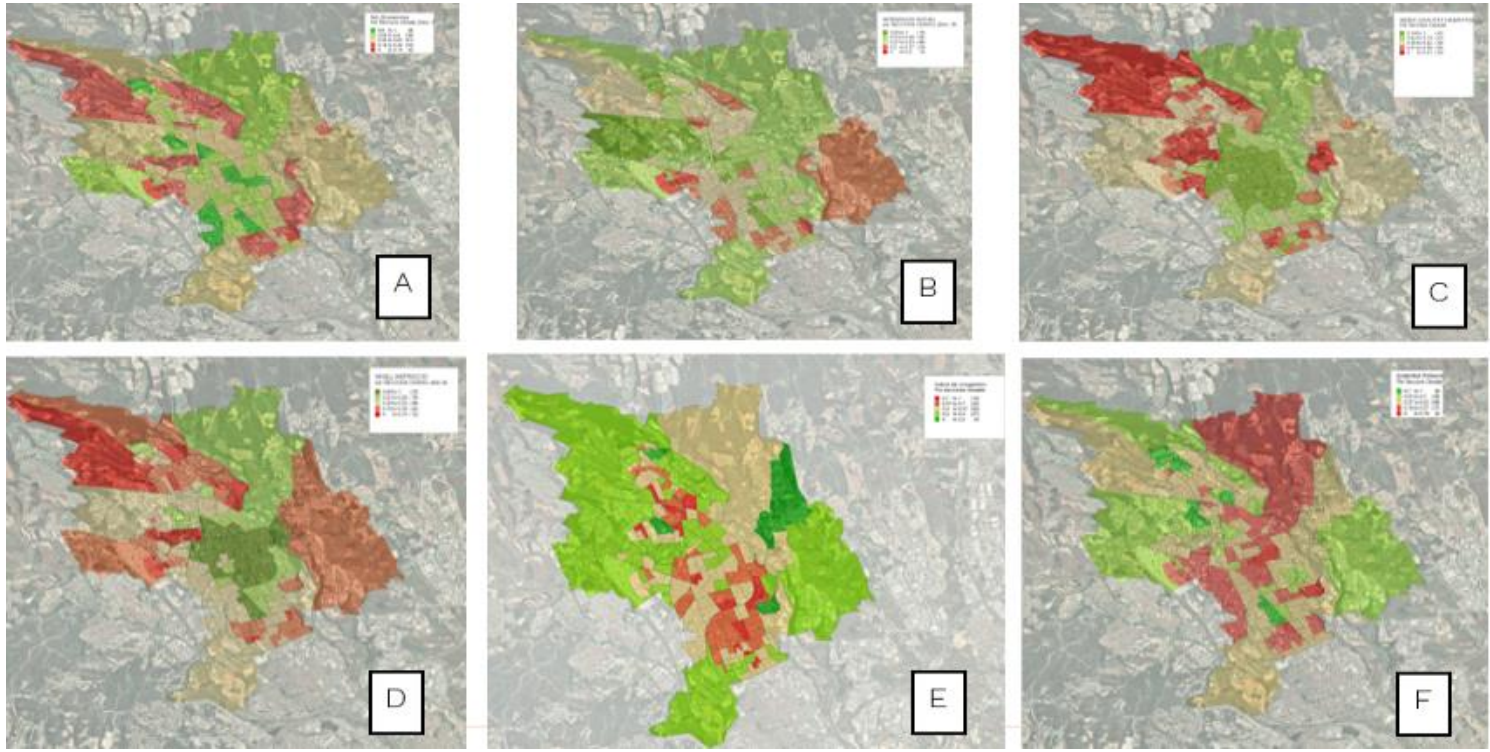

A: Actividad económica; B: integración social; C: Calidad de la vivienda; D: Nivel de instrucción; E: Congestión; F: Estabilidad de la población.

Fuente: Elaboración propia.

\subsection{Adjudicación del valor. Función de valor}

Llegado este punto y con el objeto de hacer comparables los valores obtenidos y unificar las escalas, se hace necesario normalizarlas. Esta operación se hizo a partir de la función derivada de la metodología MIVES, como se explicó anteriormente. Su forma de "S" detecta el incremento en los valores centrales, es decir en los valores alejados del rango de valor mínimo y máximo en donde se refleja una homogeneización de los valores. Este valor representa "la necesidad" de mejora de un indicador en una zona concreta y servirá para corregir, en la zona de estudio, los pesos asignados por los expertos de manera más genérica.

Una vez normalizados los valores mediante esta función, dichas secciones pasaron a tener valores 0 y 1. De manera que las secciones con los niveles de integración social más altos obtienen valores cercanos a 0 , representativos de la no necesidad de mejorar dicho indicador en la zona. De forma contraria las secciones con el valor de integración más bajo pasan a tener un indicador 1 indicativo de la necesidad de corrección del indicador. La media de los valores fue 0,4 para todas las secciones. La sección de estudio obtuvo un valor de 0,15 por debajo de la necesidad media de mejorar este indicador. Hecho que confirma que la zona no tiene grandes problemas de integración.

Al aplicar la función vemos que, de alguna manera lo que se está haciendo es "exagerar" los valores para las secciones con los valores más alejados de la media, lo cual permite identificar las necesidades en la zona de manera más clara, además las secciones cercanas sufren una cierta homogeneización (Figura 6). Los valores medios se mantienen como están mientras que los ligeramente bajos y altos se exageran permitiendo una mejor identificación y homogeneización de las zonas vecinas.

ACE, 16 (47) CC BY-ND 3.0 ES | UPC Barcelona, España | Propuesta metodológica para la determinación del mejor uso en edificios obsoletos de tejidos urbanos consolidados. DOI: http://dx.doi.org/10.5821/ace.16.47.10448 


\section{ACE Architecture, City and Environment}

E-ISSN 1886-4805

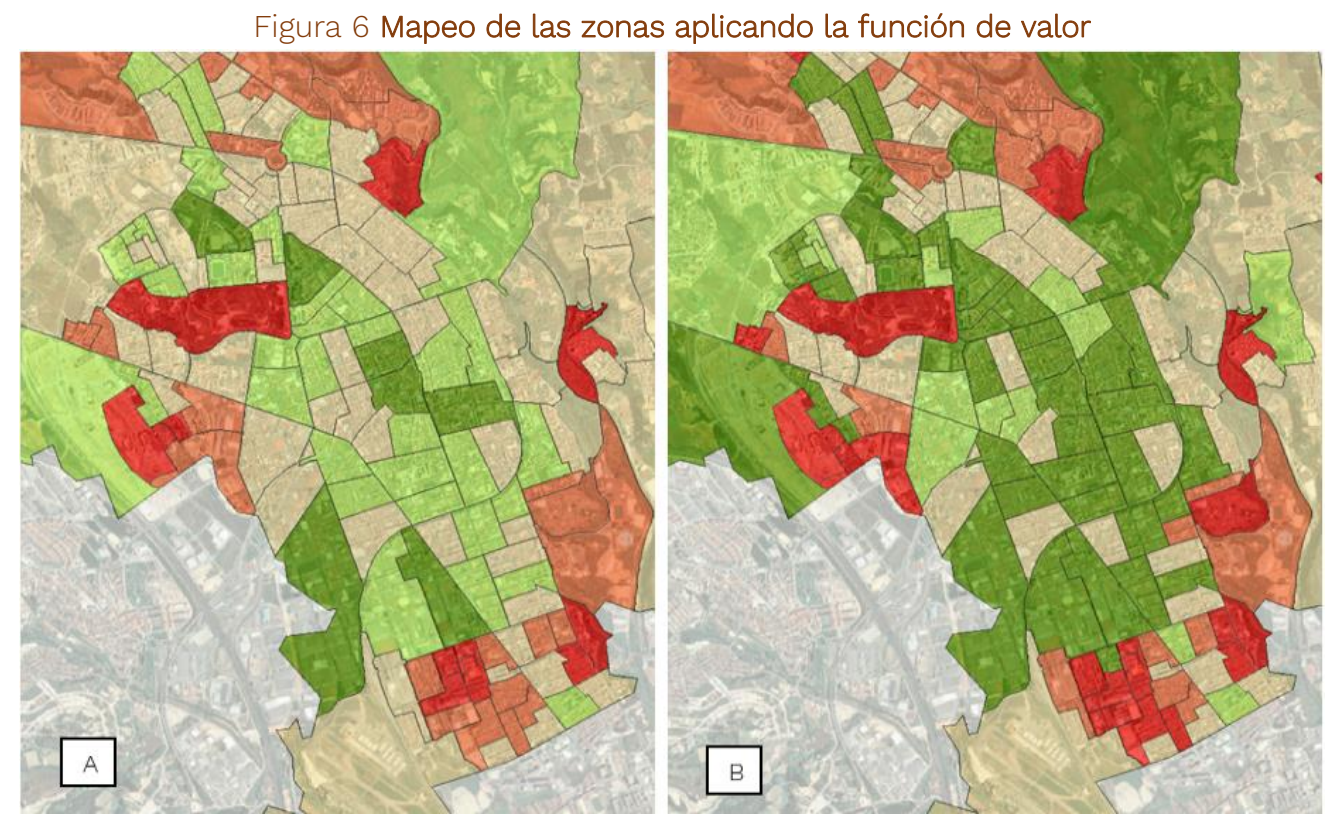

El mapa A representa los valores del indicador de actividad económica en comparación con el resto del municipio. Zonas poco homogéneas. El mapa B representa la necesidad de mejorar el indicador de actividad económica con respecto al resto del municipio.

Fuente: Elaboración propia.

\subsection{Construcción del modelo jerárquico}

Una vez obtenidos los indicadores operativos y generados los valores para cada indicador se procedió a la construcción del modelo jerárquico de acuerdo con los pasos descritos en la metodología. Las alternativas posibles se escogieron, como se ha comentado, a partir de las características tipológicas del edificio y los equipamientos existentes en la zona. De dicho análisis resultaron las siguientes: (a) Centro de Salud (CAP); (b) Alberge juvenil; (c) Museo/sala de Exposiciones; (d) Zona verde y aparcamiento; (e)Viviendas de Protección oficial. En cualquier caso, estas alternativas podrían variar, tanto en número como en tipo, pues finalmente es el decisor público el que debe establecer posibles usos a evaluar.

Para su ponderación se seleccionó un grupo de tres expertos del Centro de Política del Suelo y Valoraciones (CPSV). A cada uno de ellos se le plantea, mediante un cuestionario, dos ejercicios:

- Asignación del grado de importancia que otorga a cada uno de los distintos indicadores de calidad, respecto a tres criterios generales: ambiental, económico y social. La cuestión para resolver fue que consideraran el peso de cada indicador por comparaciones pareadas, respecto al criterio principal. Se debía ponderar, por ejemplo, la importancia de la mejora en la integración social respecto a la mejora en la actividad económica, respecto a un criterio de política social.

- Asignación del grado de importancia que otorga a la implantación de cada uso (alternativa) propuesto respecto a la mejora de cada indicador de calidad. Así al comparar un centro de salud con un alberge juvenil, respecto a la mejora en la actividad económica, el experto debería ponderar el peso del primero respecto al segundo (en este caso el alberge parece que obtendría mayor puntuación, en tanto que podría atraer cierto turismo y actividad a la zona, sin embargo, el centro de Salud también podría generar cierta actividad en tanto que generador de empleo y posible comercio en los alrededores).

ACE, 16 (47) CC BY-ND 3.0 ES | UPC Barcelona, España | Propuesta metodológica para la determinación del mejor uso en edificios obsoletos de tejidos urbanos consolidados. DOI: http://dx.doi.org/10.5821/ace.16.47.10448 
Tal y como se comentó en la metodología la escala propuesta fue la de Saaty (1987).

\subsection{Resultados}

Las respuestas obtenidas en el primer nivel jerárquico (cuestionario 1) se resumen a continuación (Tabla 3):

Tabla 3. Ponderación de cada indicador respecto a los tres criterios generales (cuestionario 1)

\begin{tabular}{|c|c|c|c|}
\hline \multirow[t]{7}{*}{1} & MEJORA ECONÓMICA & Estimación del experto & Pesos \\
\hline & Actividad Económica & 9,4 & 0,24 \\
\hline & Integración Social & 6,1 & 0,15 \\
\hline & Calidad de la vivienda & 5,9 & 0,15 \\
\hline & Nivel de instrucción & 7 & 0,18 \\
\hline & Mejora en la congestión & 5,3 & 0,13 \\
\hline & Estabilidad población & 6 & 0,15 \\
\hline \multirow[t]{7}{*}{2} & MEJORA SOCIAL & & \\
\hline & Actividad Económica & 6,3 & 0,16 \\
\hline & Integración Social & 8,7 & 0,21 \\
\hline & Calidad de la vivienda & 7,5 & 0,19 \\
\hline & Nivel de instrucción & 8 & 0,20 \\
\hline & Mejora en la congestión & 3,2 & 0,08 \\
\hline & Estabilidad población & 6,8 & 0,17 \\
\hline \multirow[t]{7}{*}{3} & MEJORA AMBIENTAL & & \\
\hline & Actividad Económica & 3,5 & 0,10 \\
\hline & Integración Social & 4,8 & 0,13 \\
\hline & Calidad de la vivienda & 7,5 & 0,21 \\
\hline & Nivel de instrucción & 6,5 & 0,18 \\
\hline & Mejora en la congestión & 8,7 & 0,24 \\
\hline & Estabilidad población & 4,8 & 0,13 \\
\hline
\end{tabular}

Fuente: Elaboración propia.

Tal y como se observa las estimaciones son coherentes con los indicadores evaluados para cada criterio general. De igual modo sucede en el segundo nivel jerárquico (Tabla 4).

Tabla 4. Ponderación de cada alternativa respecto a cada indicador compuesto (cuestionario 2)

\begin{tabular}{|l|l|l|}
\hline Actividad Económica & Estimación del experto & Pesos \\
\hline Centro de Salud (CAP) & 7,4 & 0,28 \\
\hline Alberge juvenil & 6,2 & 0,23 \\
\hline Museo/sala de Exposiciones & 6,1 & 0,23 \\
\hline Zona verde y aparcamiento & 2,9 & 0,11 \\
\hline Viviendas de Protección Oficial & 3,9 & 0,15 \\
\hline Integración Social & \multicolumn{2}{|l|}{} \\
\hline Centro de Salud (CAP) & 6,5 & 0,20 \\
\hline Alberge juvenil & 5,4 & 0,17 \\
\hline Museo/sala de Exposiciones & 6,2 & 0,19 \\
\hline Zona verde y aparcamiento & 6,3 & 0,19 \\
\hline Viviendas de Protección Oficial & 8,2 & 0,25 \\
\hline Calidad de la vivienda & \multicolumn{2}{|l|}{} \\
\hline Centro de Salud (CAP) & 5,7 & 0,20 \\
\hline Alberge juvenil & 3,2 & 0,11 \\
\hline Museo/sala de Exposiciones & 5,1 & 0,18 \\
\hline Zona verde y aparcamiento & 5,9 & 0,21 \\
\hline Viviendas de Protección Oficial & 8 & 0,29 \\
\hline Nivel de instrucción & 4,1 & 0,17 \\
\hline Centro de Salud (CAP) & 3,8 & 0,16 \\
\hline Alberge juvenil & 8,2 & 0,16 \\
\hline Museo/sala de Exposiciones & 4 & \multicolumn{2}{|l|}{} \\
\hline Zona verde y aparcamiento & \multicolumn{2}{|l|}{} \\
\hline
\end{tabular}




\begin{tabular}{|l|l|l|}
\hline \multicolumn{2}{|l|}{ Viviendas de Protección Oficial } & \multicolumn{2}{l|}{0} & 0,18 \\
\hline \multicolumn{2}{|l|}{ Mejora en la congestión } & \multicolumn{2}{l|}{} \\
\hline Centro de Salud (CAP) & 2,7 & 0,12 \\
\hline Alberge juvenil & 3,6 & 0,16 \\
\hline Museo/sala de Exposiciones & 3 & 0,13 \\
\hline Zona verde y aparcamiento & 8,6 & 0,38 \\
\hline Viviendas de Protección Oficial & 4,5 & 0,20 \\
\hline Estabilidad población & 7,4 & 0,28 \\
\hline Centro de Salud (CAP) & 3,7 & 0,14 \\
\hline Alberge juvenil & 4,4 & 0,16 \\
\hline Museo/sala de Exposiciones & 5,1 & 0,19 \\
\hline Zona verde y aparcamiento & 6,3 & 0,23 \\
\hline Viviendas de Protección Oficial & & \\
\hline
\end{tabular}

Fuente: Elaboración propia.

Cabe destacar que todas las alternativas, como ocurre en la valoración anterior, tienen un cierto peso en todos los criterios de valoración, este hecho dificulta la alternancia de los resultados, al variar los pesos en el nivel jerárquico superior. Los pesos así obtenidos representan el vector de prioridades de cada indicador de calidad para cada uno de los criterios generales (ambiental, económico y social), y el vector de prioridades de cada alternativa (usos posibles) para cada indicador. La resolución de la matriz de evaluación se muestra a continuación (Tabla 5)

Tabla 5. Resolución de la matriz de evaluación

\begin{tabular}{|c|c|c|c|c|c|c|c|c|c|c|}
\hline ECONÓMICO & iEcon & ilnt & iCal & iForm & iCong & iEst & \multirow{7}{*}{$x$} & & \multirow{7}{*}{$=$} & PESOS \\
\hline Centro de Salud (CAP) & 0,28 & 0,20 & 0,20 & 0,17 & 0,12 & 0,28 & & 0,24 & & 0,2144 \\
\hline Alberge juvenil & 0,23 & 0,17 & 0,11 & 0,16 & 0,16 & 0,14 & & 0,15 & & 0,1676 \\
\hline Museo/sala de Exposiciones & 0,23 & 0,19 & 0,18 & 0,34 & 0,13 & 0,16 & & 0,15 & & 0,2127 \\
\hline Zona verde y aparcamiento & 0,11 & 0,19 & 0,21 & 0,16 & 0,38 & 0,19 & & 0,18 & & 0,1958 \\
\hline \multirow[t]{2}{*}{ Viviendas de Protección Oficial } & 0,15 & 0,25 & 0,29 & 0,18 & 0,20 & 0,23 & & \multirow{2}{*}{\begin{tabular}{|l|}
0,13 \\
0,15 \\
\end{tabular}} & & \multirow[t]{2}{*}{0,2094} \\
\hline & & & & & & & & & & \\
\hline \multicolumn{11}{|l|}{ SOCIAL } \\
\hline Centro de Salud (CAP) & 0,28 & 0,20 & 0,20 & 0,17 & 0,12 & 0,28 & \multirow{6}{*}{$x$} & 0,16 & \multirow{6}{*}{$=$} & 0,2130 \\
\hline Alberge juvenil & 0,23 & 0,17 & 0,11 & 0,16 & 0,16 & 0,14 & & 0,21 & & 0,1598 \\
\hline Museo/sala de Exposiciones & 0,23 & 0,19 & 0,18 & 0,34 & 0,13 & 0,16 & & 0,19 & & 0,2149 \\
\hline Zona verde y aparcamiento & 0,11 & 0,19 & 0,21 & 0,16 & 0,38 & 0,19 & & 0,20 & & 0,1922 \\
\hline \multirow[t]{2}{*}{ Viviendas de Protección Oficial } & 0,15 & 0,25 & 0,29 & 0,18 & 0,20 & 0,23 & & \multirow{2}{*}{\begin{tabular}{|l|}
0,08 \\
0,17 \\
\end{tabular}} & & \multirow[t]{2}{*}{0,2200} \\
\hline & & & & & & & & & & \\
\hline \multicolumn{11}{|l|}{ AMBIENTAL } \\
\hline Centro de Salud (CAP) & 0,28 & 0,20 & 0,20 & 0,17 & 0,12 & 0,28 & \multirow{6}{*}{$x$} & 0,10 & \multirow{6}{*}{$=$} & 0,1935 \\
\hline Alberge juvenil & 0,23 & 0,17 & 0,11 & 0,16 & 0,16 & 0,14 & & 0,13 & & 0,1549 \\
\hline Museo/sala de Exposiciones & 0,23 & 0,19 & 0,18 & 0,34 & 0,13 & 0,16 & & 0,21 & & 0,2018 \\
\hline Zona verde y aparcamiento & 0,11 & 0,19 & 0,21 & 0,16 & 0,38 & 0,19 & & 0,18 & & 0,2294 \\
\hline Viviendas de Protección Oficial & 0,15 & 0,25 & 0,29 & 0,18 & 0,20 & 0,23 & & 0,24 & & 0,2204 \\
\hline & & & & & & & & 0,13 & & \\
\hline
\end{tabular}

Fuente: Elaboración propia.

Así pues, con los pesos valorados de manera general, sin tener en cuenta la zona de estudio, y de acuerdo con la importancia de cada indicador y criterio valorados por el grupo de expertos, el centro de Salud es la mejor alternativa desde un punto de vista Económico. Las Viviendas de Protección oficial son la mejor alternativa desde un punto de vista social, y finalmente la zona verde y aparcamiento resultó ser la mejor alternativa desde un criterio ambiental. El paso siguiente en la aplicación del método AHP (cuarto paso), consistiría en interactuar nuevamente, pero ahora en el tercer nivel jerárquico (ambiental, económico y social). Para ello, tendría que determinarse una relación al confrontar cada uso con cada criterio principal. Esta relación es la que debería estimar el decisor público en función de si quiere aplicar una política ambiental, económica o social. Se muestran a continuación ejemplo de dos ponderaciones (Tabla 6)

ACE, 16 (47) CC BY-ND 3.0 ES | UPC Barcelona, España | Propuesta metodológica para la determinación del mejor uso en edificios obsoletos de tejidos urbanos consolidados. DOI: $\underline{\text { http://dx.doi.org/10.5821/ace.16.47.10448 }}$ 
Tabla 6 Ejemplo resoluciones de la matriz en el tercer nivel jerárquico

\begin{tabular}{|c|c|c|c|c|c|c|c|c|}
\hline & Económico & Social & Ambiental & \multirow{5}{*}{$x$} & & \multirow{5}{*}{\begin{tabular}{|l|} 
Económico \\
Social \\
Ambiental \\
\end{tabular}} & \multirow{5}{*}{$=$} & \multirow{5}{*}{\begin{tabular}{|l|} 
Prioridades \\
0,2016 \\
0,1584 \\
0,2066 \\
0,2153 \\
\end{tabular}} \\
\hline Centro de Salud (CAP) & 0,2144 & 0,2130 & 0,1935 & & \multirow{4}{*}{$\begin{array}{l}0,2 \\
0,2 \\
0,6\end{array}$} & & & \\
\hline Alberge juvenil & 0,1676 & 0,1598 & 0,1549 & & & & & \\
\hline Museo/sala de Exposiciones & 0,2127 & 0,2149 & 0,2018 & & & & & \\
\hline Zona verde y aparcamiento & 0,1958 & 0,1922 & 0,2294 & & & & & \\
\hline & & & & & & & & \\
\hline $\begin{array}{l}\text { Viviendas de Protección } \\
\text { Oficial }\end{array}$ & 0,2094 & 0,2200 & 0,2204 & & & & & 0,2181 \\
\hline Centro de Salud (CAP) & 0,2144 & 0,2130 & 0,1935 & & 1 & Económico & & 0,2144 \\
\hline Alberge juvenil & 0,1676 & 0,1598 & 0,1549 & & 0 & Social & - & 0,1676 \\
\hline Museo/sala de Exposiciones & 0,2127 & 0,2149 & 0,2018 & & 0 & Ambiental & & 0,2127 \\
\hline Zona verde y aparcamiento & 0,1958 & 0,1922 & 0,2294 & & & & & 0,1958 \\
\hline $\begin{array}{l}\text { Viviendas de Protección } \\
\text { Oficial }\end{array}$ & 0,2094 & 0,2200 & 0,2204 & & & & & 0,2094 \\
\hline
\end{tabular}

Fuente: Elaboración propia.

La primera parte de la tabla muestra una priorización de la política ambiental con 60\% y el resto por igual al 20\%. La alternativa Viviendas de protección, zona verde y aparcamiento, y museo, resultan por este orden las mejor valoradas. La segunda parte de la tabla muestra un ejemplo de priorización de política estrictamente económica (pesos 1,0,0) la mejor alternativa seria entonces el centro de salud seguido por el museo o sala de exposiciones.

\subsection{Corrección de los pesos}

Las prioridades obtenidas una vez concluido este procedimiento se refieren a un caso general, por el desconocimiento del experto sobre la zona, tal y como se ha explicado anteriormente. Para adaptar los pesos de los indicadores a la realidad social se multiplicaron por el valor otorgado por la función para la sección censal objeto de estudio (Tabla 7). De manera que el producto resultante representaba la prioridad de cada alternativa para la zona (producto de la ponderación de los expertos y el valor asignado por la función). Por ejemplo, en general la mejora de la actividad económica tiene el mayor peso desde un punto de vista de la promoción de la política económica, pero puede resultar que la zona de estudio ya muestre valores altos de actividad económica con respecto al municipio, con lo cual se multiplicará por valores cercanos a 0 .

Tabla 7 Valores de "necesidad" de la zona de estudio asignados por la función de valor

\begin{tabular}{|l|l|l|l|l|l|l|l|}
\hline & $\begin{array}{l}\text { Actividad } \\
\text { Económica }\end{array}$ & $\begin{array}{l}\text { Integración } \\
\text { Social }\end{array}$ & $\begin{array}{l}\text { Calidad de la } \\
\text { vivienda }\end{array}$ & $\begin{array}{l}\text { Nivel de } \\
\text { instrucción }\end{array}$ & $\begin{array}{l}\text { Mejora en la } \\
\text { congestión }\end{array}$ & $\begin{array}{l}\text { Estabilidad } \\
\text { población }\end{array}$ \\
\hline Necesidad & 0,0571 & 0,1513 & 0,1494 & 0,6346 & 0,7566 & 0,3971 \\
\hline
\end{tabular}

Fuente: Elaboración propia.

Es decir, en relación con el resto del municipio, la zona de estudio requiere de mejoras en el nivel de instrucción y en la congestión. De manera que al corregir los pesos iniciales las mejoras en estos indicadores parecen ser ahora los más importantes, de acuerdo con la siguiente tabla (Tabla 8):

Tabla 8 Corrección de los pesos generales por las características de la zona de estudio

\begin{tabular}{|c|c|c|c|c|c|c|c|c|}
\hline \multirow[t]{7}{*}{1} & ECONÓMICO & $\begin{array}{l}\text { Estimación } \\
\text { del experto }\end{array}$ & Pesos & & $\begin{array}{l}\text { Valor Zc } \\
\text { estudio }\end{array}$ & & & Pesos corregidos \\
\hline & Actividad Económica & 9,4 & 0,24 & \multirow{6}{*}{$x$} & 0,0571 & \multirow{6}{*}{$=$} & 0,013521 & 0,0407 \\
\hline & Integración Social & 6,1 & 0,15 & & 0,1513 & & 0,023241 & 0,0700 \\
\hline & Calidad de la vivienda & 5,9 & 0,15 & & 0,1494 & & 0,022197 & 0,0669 \\
\hline & Nivel de instrucción & 7 & 0,18 & & 0,6346 & & 0,111893 & 0,3372 \\
\hline & Mejora en la congestión & 5,3 & 0,13 & & 0,7566 & & 0,101012 & 0,3044 \\
\hline & Estabilidad población & 6 & 0,15 & & 0,3971 & & 0,060016 & 0,1808 \\
\hline
\end{tabular}

ACE, 16 (47) CC BY-ND 3.0 ES | UPC Barcelona, España | Propuesta metodológica para la determinación del mejor uso en edificios obsoletos de tejidos urbanos consolidados. DOI: http://dx.doi.org/10.5821/ace.16.47.10448 


\begin{tabular}{|c|c|c|c|c|c|c|c|c|}
\hline \multirow[t]{2}{*}{2} & \multicolumn{8}{|l|}{ SOCIAL } \\
\hline & Actividad Económica & 6,3 & 0,16 & \multirow{6}{*}{$\mathrm{x}$} & 0,0571 & & 0,008883 & 0,0277 \\
\hline & Integración Social & 8,7 & 0,21 & & 0,1513 & & 0,032492 & 0,1013 \\
\hline & Calidad de la vivienda & 7,5 & 0,19 & & 0,1494 & & 0,027659 & 0,0862 \\
\hline & Nivel de instrucción & 8 & 0,20 & & 0,6346 & & 0,125352 & 0,3907 \\
\hline & Mejora en la congestión & 3,2 & 0,08 & & 0,7566 & & 0,059784 & 0,1863 \\
\hline & Estabilidad población & 6,8 & 0,17 & & 0,3971 & & 0,066674 & 0,2078 \\
\hline \multirow[t]{7}{*}{3} & AMBIENTAL & & & & & & & \\
\hline & Actividad Económica & 3,5 & 0,10 & \multirow{6}{*}{$x$} & 0,0571 & & 0,005583 & 0,0136 \\
\hline & Integración Social & 4,8 & 0,13 & & 0,1513 & & 0,02028 & 0,0495 \\
\hline & Calidad de la vivienda & 7,5 & 0,21 & & 0,1494 & & 0,03129 & 0,0764 \\
\hline & Nivel de instrucción & 6,5 & 0,18 & & 0,6346 & & 0,11522 & 0,2814 \\
\hline & Mejora en la congestión & 8,7 & 0,24 & & 0,7566 & & 0,183876 & 0,4490 \\
\hline & Estabilidad población & 4,8 & 0,13 & & 0,3971 & & 0,053243 & 0,1300 \\
\hline
\end{tabular}

Fuente: Elaboración propia.

Como se puede observar el peso de los indicadores varía al ser corregido por las necesidades de la zona de estudio. Al volver a resolver la matriz con los nuevos pesos se obtienen los siguientes resultados (Tabla 9 ).

Tabla 9 Resolución de la matriz con los pesos corregidos

\begin{tabular}{|c|c|c|c|c|c|c|c|c|c|c|}
\hline ECONÓMICO & Econ & ilnt & iCal & iForm & iCong & iEst & \multirow{6}{*}{$\mathrm{x}$} & & & PESOS \\
\hline Centro de Salud (CAP) & 0,28 & 0,20 & 0,20 & 0,17 & 0,12 & 0,28 & & 0,0407 & & 0,1821 \\
\hline Alberge juvenil & 0,23 & 0,17 & 0,11 & 0,16 & 0,16 & 0,14 & & 0,0700 & & 0,1551 \\
\hline Museo/sala de Exposiciones & 0,23 & 0,19 & 0,18 & 0,34 & 0,13 & 0,16 & & 0,0669 & & 0,2186 \\
\hline Zona verde y aparcamiento & 0,11 & 0,19 & 0,21 & 0,16 & 0,38 & 0,19 & & 0,3372 & & 0,2385 \\
\hline Viviendas de Protección Oficial & 0,15 & 0,25 & 0,29 & 0,18 & 0,20 & 0,23 & & 0,3044 & & 0,2057 \\
\hline & & & & & & & & 0,1808 & & \\
\hline SOCIAL & Econ & ilnt & iCal & iForm & iCong & iEst & \multirow{6}{*}{$x$} & ens & & \multirow{7}{*}{\begin{tabular}{|l|}
0,1908 \\
0,1525 \\
0,2316 \\
0,2158 \\
0,2092 \\
\end{tabular}} \\
\hline Centro de Salud (CAP) & 0,28 & 0,20 & 0,20 & 0,17 & 0,12 & 0,28 & & 0,0277 & \multirow{5}{*}{$=$} & \\
\hline Alberge juvenil & 0,23 & 0,17 & 0,11 & 0,16 & 0,16 & 0,14 & & 0,1013 & & \\
\hline Museo/sala de Exposiciones & 0,23 & 0,19 & 0,18 & 0,34 & 0,13 & 0,16 & & 0,0862 & & \\
\hline Zona verde y aparcamiento & 0,11 & 0,19 & 0,21 & 0,16 & 0,38 & 0,19 & & 0,3907 & & \\
\hline Viviendas de Protección Oficial & 0,15 & 0,25 & 0,29 & 0,18 & 0,20 & 0,23 & & 0,1863 & & \\
\hline & & & & & & & & 0,2078 & & \\
\hline AMBIENTAL & Econ & ilnt & iCal & iForm & iCong & iEst & \multirow{6}{*}{$x$} & & & \\
\hline Centro de Salud (CAP) & 0,28 & 0,20 & 0,20 & 0,17 & 0,12 & 0,28 & & 0,0136 & \multirow{5}{*}{$=$} & 0,1665 \\
\hline Alberge juvenil & 0,23 & 0,17 & 0,11 & 0,16 & 0,16 & 0,14 & & 0,0495 & & 0,1540 \\
\hline Museo/sala de Exposiciones & 0,23 & 0,19 & 0,18 & 0,34 & 0,13 & 0,16 & & 0,0764 & & 0,2025 \\
\hline Zona verde y aparcamiento & 0,11 & 0,19 & 0,21 & 0,16 & 0,38 & 0,19 & & 0,2814 & & 0,2704 \\
\hline Viviendas de Protección Oficial & 0,15 & 0,25 & 0,29 & 0,18 & 0,20 & 0,23 & & 0,4490 & & 0,2066 \\
\hline
\end{tabular}

Fuente: elaboración propia

Como puede observarse la importancia de las alternativas ha variado en función de las necesidades de la zona de estudio. Finalmente, al ponderar la importancia de los criterios generales (social, económico y social), se obtiene la mejor alternativa para la zona de estudio. Los pesos considerados en este caso fueron: $60 \%$ ambiental, $20 \%$ económico, y 20\% social (Tabla 10):

Tabla 10. Obtención mejor alternativa según criterios generales (social, económico y social)

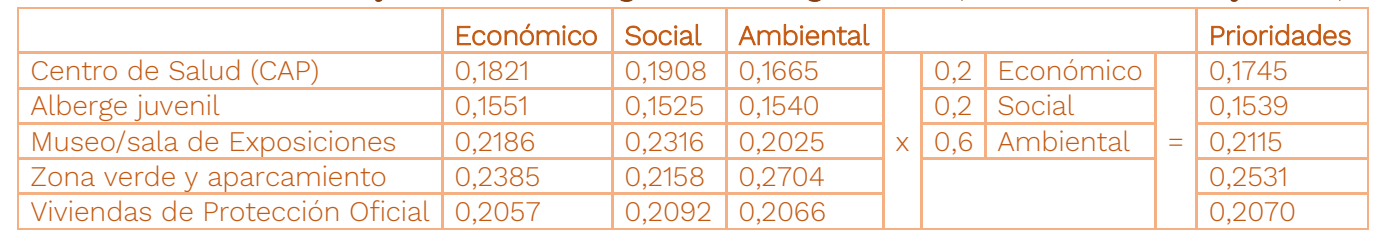

Fuente: Elaboración propia. 
Así pues, utilizando los mismos pesos que en el caso general, la zona verde y aparcamiento, de acuerdo con los déficits de la zona, es la mejor alternativa con un 25\% de importancia sobre el resto. Si los criterios generales se alteran en este tercer nivel jerárquico se obtienen las siguientes alternativas (Tabla 11):

Tabla 11. Resumen de las alternativas alternando los criterios generarles

\begin{tabular}{|l|l|l|l|}
\hline Económico & 0 & 1 & 0 \\
\hline Social & 0 & 0 & 1 \\
\hline Ambiental & 1 & 0 & 0 \\
\hline & $\begin{array}{l}\text { Zona verde y } \\
\text { aparcamiento (27\%) }\end{array}$ & $\begin{array}{l}\text { Zona verde y } \\
\text { aparcamiento (23\%) }\end{array}$ & $\begin{array}{l}\text { Museo y sala de } \\
\text { exposiciones (23\%) }\end{array}$ \\
\hline
\end{tabular}

Fuente: Elaboración propia.

Es decir, independientemente de la importancia que se les otorgue a los criterios generales, las únicas alternativas posibles de entre las consideradas en la zona de estudio son la zona verde/aparcamiento y el museo/sala de exposiciones. Esto es así porque son las alternativas mejor valoradas en la mejora en la congestión y en el nivel de instrucción, que a su vez son los déficits más importantes en la zona, en comparación con el resto del municipio.

Por otro lado, tal y como se ha mostrado, cuando las alternativas a valorar tienen una cierta importancia en todos los criterios de comparación, es decir no son completamente independientes entre ellas, ocurre que a medida que se aumenta el nivel de jerarquía, la importancia de los criterios tiende a 0 . Esto es, los pesos asignados en los niveles inferiores resultan ser determinantes en la elección de la mejor alternativa, mientras que los pesos asignados en los niveles superiores disminuyen en importancia llegando a no afectar finalmente a las diferentes alternativas, que "arrastran" pesos para cada uno de los criterios. Además, se observa que cuando las secciones censales son deficitarias en varios indicadores, tendrán siempre mayor necesidad de implantación de los nuevos usos.

\section{Conclusiones}

En el caso presentado, parece que a partir de la combinación de los métodos de análisis (ACP y AHP) es posible establecer un uso óptimo para una zona concreta de una forma más consistente. Sin embargo, al caracterizar la zona, la interpretación de los factores, tanto el signo como la magnitud de las correlaciones entre variables, no siempre es fácil, y resultará de vital importancia el conocimiento que el experto tenga sobre la materia de investigación.

De los resultados obtenidos se desprende que cuando las alternativas a valorar tienen una cierta importancia en todos los criterios de comparación, es decir no son completamente independientes entre ellas, ocurre que a medida que se aumenta el nivel de jerarquía, la importancia de los criterios tiende a 0 . De manera que, en el caso descrito, sería conveniente buscar otras alternativas que mejoraran los puntos débiles de las propuestas en el caso de estudio. La escasa diferencia entre las prioridades (pesos) obtenidas por cada una de ellas hace que la clasificación propuesta resulte cuestionable, cuando no arbitraria, perdiendo solidez, en tanto que la evaluación de las posibles actuaciones depende demasiado de la percepción del experto.

ACE, 16 (47) CC BY-ND 3.0 ES | UPC Barcelona, España | Propuesta metodológica para la determinación del mejor uso en edificios obsoletos de tejidos urbanos consolidados. DOI: http://dx.doi.org/10.5821/ace.16.47.10448 
Respecto a la actuación en núcleos urbanos consolidados, las concentraciones urbanas son sistemas complejos en donde las situaciones ambientales, sociales, y económicas pueden presentar diferencias significativas entre las distintas zonas. Desarrollar metodologías de actuación, en tejidos urbanos consolidados, con niveles altos de desagregación (a nivel censal, por ejemplo) proporcionaría a los responsables políticos un instrumento mediante el cual la información, presentada de manera concisa y representativa, podría ser usada fácilmente. Dicha construcción de información adecuada y actualizada ha de permitir, a los tomadores de decisiones, diseñar y priorizar la ejecución de estrategias que impacten de forma diferencial en las distintas zonas de la ciudad de forma más eficiente y racional.

Del mismo modo, en futuros trabajos, podría construirse un mapa, por secciones censales indicativo de la necesidad de la implantación para cada uso de los planteados y comparase con otros municipios y casos de estudio, siempre que se disponga de una base de datos comparable. Igualmente podrían compararse resultados con otras metodologías existentes parecidas, no tan complejas. La novedad en este caso radica en la incorporación del análisis de componentes principales para la definición de los criterios a aplicar, según las necesidades de cada zona, y el uso particular de Delphi para la obtención de opiniones fiables de los expertos.

\section{Autoría}

El trabajo expuesto surge de la investigación realizada por el primer autor en el marco de la tesis de fin de máster realizada por él, dentro del programa de doctorado de Gestión y Valoración Urbana y Arquitectónica. El segundo autor, como director de tesis, ha revisado y completado su contenido.

Conflicto de intereses: Los autores declaran que no hay conflicto de intereses.

\section{Bibliografía}

Aguarón, J., \& Moreno-Jiménez, J. M. (2003). The geometric consistency index: Approximated thresholds. European Journal of Operational Research, 147(1), 137-145. DOI: 10.1016/S0377$\underline{2217(02) 00255-2}$

Anderson, T. W. (1984). Introduction to multivariate statistical analysis (2nd ed.).

Azqueta Oyarzun, D., \& Delacamara Andrés, G. (2004). El papel de las variables ambientales en la nueva geografía económica. Investigaciones Regionales, primavera, 145-175. http://www.redalyc.org/src/inicio/ArtPdfRed.jsp?iCve=28900408

Bagočius, V., Zavadskas, E. K., \& Turskis, Z. (2014). Multi-person selection of the best wind turbine based on the multi-criteria integrated additive-multiplicative utility function. Journal of Civil Engineering and Management, 20(4), 590-599. DOI: 10.3846/13923730.2014.932836

Barba-Romero, S. (1987). Panorámica actual de la decisión multicriterio discreta. Investigaciones Economicas, 11(2), 279-308. https://econpapers.repec.org/RePEc:iec:inveco:v:11:y:1987:i:2:p:279-308

Brans, J.-P., \& Mareschal, B. (2005). Promethee Methods. In Multiple Criteria Decision Analysis: State of the Art Surveys (pp. 163-186). Springer-Verlag. DOI: $\underline{10.1007 / 0-387-23081-55}$ 
Brans, J. P., Vincke, P., \& Mareschal, B. (1986). How to select and how to rank projects: The Promethee method. European Journal of Operational Research, 24(2), 228-238. DOI: 10.1016/0377-2217(86)90044$\underline{5}$

Buchanan, J., Sheppard, P., \& Vanderpooten, D. (1999). Project ranking using Electre III.

Crama, Y., \& Hansen, P. (1983). An Introduction to the Electre Research Programme. In Essays and Surveys on Multiple Criteria Decision Making (pp. 31-42). DOI: 10.1007/978-3-642-46473-7_4

Crawford, G., \& Williams, C. (1985). A note on the analysis of subjective judgment matrices. Journal of Mathematical Psychology, 29(4), 387-405. DOI: 10.1016/0022-2496(85)90002-1

Dalkey, N., \& Helmer, O. (1963). An Experimental Application of the DELPHI Method to the Use of Experts. Management Science, 9(3), 458-467. DOI: 10.1287/mnsc.9.3.458

Davies, A. L., Bryce, R., \& Redpath, S. M. (2013). Use of Multicriteria Decision Analysis to Address Conservation Conflicts. Conservation Biology, 27(5), 936-944. DOI: 10.1111/cobi.12090

Debreu, G. (1959). Topological Methods in Cardinal Utility Theory (Issue 76). https://econpapers.repec.org/RePEc:cwl:cwldpp:76

Domínguez-Berjórn, M. F., \& Borrell, C. (2005). Mortalidad y privación socioeconómica en las secciones censales y los distritos de Barcelona. Gaceta Sanitaria, 19, 363-369. http://scielo.isciii.es/scielo.php?script=sci arttext\&pid=S0213-91112005000500004\&nrm=iso

Ferri, C. P., Prince, M., Brayne, C., Brodaty, H., Fratiglioni, L., Ganguli, M., Hall, K., Hasegawa, K., Hendrie, H., Huang, Y., Jorm, A., Mathers, C., Menezes, P. R., Rimmer, E., \& Scazufca, M. (2005). Global prevalence of dementia: a Delphi consensus study. The Lancet, 366(9503), 2112-2117. DOI: 10.1016/S0140$\underline{6736(05) 67889-0}$

Figueira, J., Mousseau, V., \& Roy, B. (2005). Electre Methods. In Multiple Criteria Decision Analysis: State of the Art Surveys (pp. 133-153). Springer-Verlag. DOI: 10.1007/0-387-23081-5_4

Fishburn, P. C. (1974). Lexicographic Orders, Utilities and Decision Rules: A Survey. Management Science, 20(11), 1442-1471. DOI: $\underline{10.1287 / \mathrm{mnsc} .20 .11 .1442}$

Fishburn, P. C. (1990). Utility Theory and Decision Theory. In Utility and Probability (pp. 303-312). Palgrave Macmillan UK. DOI: 10.1007/978-1-349-20568-4 40

Fisher, M. R., Churchman, C. W., Ackoff, R. L., \& Arnoff, E. L. (1959). Introduction to Operations Research. The Economic Journal, 69(276), 785. DOI: $\underline{10.2307 / 2227681}$

Frías Jiménez, R. A. (2008). Herramientas de apoyo a la solución de problemas no estructurados en empresas turísticas. Editorial Universitaria.

García Leyton, L. A. (2004). Aplicación del análisis multicriterio en la evaluación de impactos ambientales. Universitat Politècnica de Catalunya (UPC).

Gómez-López, M. D., García-Cascales, M. S., Bayo, J., \& Angosto, J. M. (2007). Utilización de técnicas de análisis multicriterio en la elección de un sistema de desinfección de agua residual. XI Congreso

ACE, 16 (47) CC BY-ND 3.0 ES | UPC Barcelona, España | Propuesta metodológica para la determinación del mejor uso en edificios obsoletos de tejidos urbanos consolidados. DOI: http://dx.doi.org/10.5821/ace.16.47.10448 
Internacional de Ingeniería de Proyectos

https://www.aeipro.com/files/congresos/2007lugo/ciip07_1112_1121.507.pdf

(pp.

1112-1121).

Hair, J. F., Anderson, R. E., \& Tatham, R. L. (1999). Analisis Multivariante. Pretince-Hall.

Johnson, D. E. (1999). Métodos multivariados aplicados al análisis de datos. Thomson Editores.

Jolliffe, I. T. (1986). Principal Components Analysis (2nd ed.). Springer-Verlag.

Josa, I., Pons, O., Fuente, A. de la, \& Aguado, A. (2020). Multi-criteria decision-making model to assess the sustainability of girders and trusses: Case study for roofs of sports halls. Journal of Cleaner Production, 249, 119312. DOI: 10.1016/j.jclepro.2019.119312

Kaoru, T. (1996). Two Technical Notes on AHP based on the Geometric Mean Method. Proceedings of the 4th International Symposium on AHP, Simon Fraser University, Canada, (pp. 375-381).

Keeney, R. L., \& Raiffa, H. (1993). Decisions with Multiple Objectives. Cambridge University Press. DOI: 10.1017/CBO9781139174084

Lamelas García, M. T. (2014). Aplicación de técnicas de análisis multicriterio a la localización óptima de extracciones de arenas y gravas en el entorno de Zaragoza. Boletín de La Asociación de Geógrafos Españoles, 66. DOI: 10.21138/bage.1778

Landeta, J. (1999). El método Delphi: una técnica de previsión del futuro. Ariel. https://books.google.es/books?id=yJWBPwAACAAJ

Medina, B., \& García, M. (2016). Aplicación de métodos de decisión multicriterio discretos al análisis de alternativas en estudios informativos de infraestructuras de transporte. Pensamiento Matemático, 6 , $27-45$.

Moher, D., Cook, D. J., Eastwood, S., Olkin, I., Rennie, D., \& Stroup, D. F. (1999). Improving the quality of reports of meta-analyses of randomised controlled trials: the QUOROM statement. The Lancet, 354(9193), 1896-1900. DOI: 10.1016/S0140-6736(99)04149-5

Moreno-Loscertales, J. M., Moreno-Jiménez, J. M., \& Turón Lanuza, A. (2019). Incorporación de aspectos intangibles en el análisis de la viabilidad del transporte urbano de Zaragoza. Estudios de Economía Aplicada, 37(1), 80. DOI: 10.25115/eea.v37i1.2572

Munda, G., Nijkamp, P., \& Rietveld, P. (1995). Qualitative multicriteria methods for fuzzy evaluation problems: An illustration of economic-ecological evaluation. European Journal of Operational Research, 82(1), 79-97. DOI: 10.1016/0377-2217(93)E0250-2

Nijkamp, P., \& Delft, A. va. (1977). Multi-Criteria Analysis and Regional Decision-Making (1st ed.). Springer US.

Oses, O. U. (2017). Propuesta Metodológica para el Desarrollo de sistemas de transporte sostenible a través del planeamiento de entornos urbanos. Universidad del País Vasco. https://addi.ehu.es/handle/10810/25650

Peters, W. S., \& Butler, J. Q. (1970). The construction of regional economic indicators by principal components. The Annals of Regional Science, 4(1), 1-14. DOI: $10.1007 /$ BF01287726

ACE, 16 (47) CC BY-ND 3.0 ES | UPC Barcelona, España | Propuesta metodológica para la determinación del mejor uso en edificios obsoletos de tejidos urbanos consolidados. DOI: $\underline{\text { http://dx.doi.org/10.5821/ace.16.47.10448 }}$ 
Pons-Valladares, O., \& Nikolic, J. (2020). Sustainable Design, Construction, Refurbishment and Restoration of Architecture: A Review. Sustainability, 12(22), 9741. DOI: 10.3390/su12229741

Romero, C. (1994). Aplicaciones de la teoría de la decisión multicriterio en la planificación de los recursos forestales. Agricultura y Sociedad $73, \quad 41-70 \quad$ (1994). https://helvia.uco.es/xmlui/handle/10396/5627\#.YCZyOYvEQhI.mendeley

Roy, B. (1968). Classement et choix en présence de points de vue multiples. Revue Française d'informatique et de Recherche Opérationnelle, 2(8), 57-75. DOI: 10.1051/ro/196802V100571

Saaty, R. W. (1987). The analytic hierarchy process-what it is and how it is used. Mathematical Modelling, 9(3-5), 161-176. DOI: 10.1016/0270-0255(87)90473-8

Saaty, T. L. (1990). How to make a decision: The analytic hierarchy process. European Journal of Operational Research, 48(1), 9-26. DOI: 10.1016/0377-2217(90)90057-I

Saaty, T. L. (1994). How to Make a Decision: The Analytic Hierarchy Process. Interfaces, 24(6), 19-43. DOI: $10.1287 /$ inte.24.6.19

Streimikiene, D., Balezentis, T., Krisciukaitienè, I., \& Balezentis, A. (2012). Prioritizing sustainable electricity production technologies: MCDM approach. Renewable and Sustainable Energy Reviews, 16(5), 3302-3311. 10.1016/j.rser.2012.02.067

Wind, Y., \& Saaty, T. L. (1980). Marketing Applications of the Analytic Hierarchy Process. Management Science, 26(7), 641-658. DOI: 10.1287/mnsc.26.7.641

Yager, R. R. (1988). On ordered weighted averaging aggregation operators in multicriteria decisionmaking. IEEE Transactions on Systems, Man, and Cybernetics, 18(1), 183-190. DOI: $\underline{10.1109 / 21.87068}$

Zavadskas, E. ., Turskis, Z., \& Bagočius, V. (2015). Multi-criteria selection of a deep-water port in the Eastern Baltic Sea. Applied Soft Computing, 26, 180-192. DOI: 10.1016/j.asoc.2014.09.019

Zavadskas, E. K., Skibniewski, M. J., \& Antucheviciene, J. (2014). Performance analysis of Civil Engineering Journals based on the Web of Science ${ }^{\circledR}$ database. Archives of Civil and Mechanical Engineering, 14(4), 519-527. DOI: 10.1016/j.acme.2014.05.008

Zeleny, M. (1974). Linear Multiobjective Programming (Vol. 95). Springer Berlin Heidelberg. DOI: 10.1007/978-3-642-80808-1 